\title{
CRACK DETECTION IN SIMPLY SUPPORTED BEAMS USING STATIONARY WAVELET TRANSFORM OF MODAL DATA
}

\author{
Shuncong Zhong ${ }^{1}$ and S Olutunde Oyadiji ${ }^{2 *}$
}

1. Department of Electrical Engineering and Electronics, The University of Liverpool, Liverpool L69 3GJ, UK

2. Dynamics and Aeroelasticity Research Group, School of Mechanical, Aerospace and Civil Engineering, The University of Manchester, M13 9PL, UK

\begin{abstract}
A new approach, based on Stationary Wavelet Transform (SWT), is proposed in this paper for crack detection in beam-like structures. SWT is different from Discrete Wavelet Transform (DWT); SWT is a redundant transform that doubles the number of input samples at each iteration, which can provide a more accurate estimation of the variances and facilitate the identification of salient features in a signal, especially for recognizing noise or signal rupture. The mode shape of a cracked beam with a small crack depth, while apparently a single smooth curve, actually exhibits a local peak or discontinuity in the region of damage. The mode shape 'signal' can be approximately considered as that of the intact beam contaminated by 'noise' which consists of response noise and the additional response due to the crack. In this way, the signal can be decomposed by SWT into a smooth curve, called approximation coefficient, and a detail coefficient curve, which includes crack information that is useful for damage detection, respectively. In this paper, the modal responses of damaged simply supported beams are computed using the finite element method. The effect of noise on the proposed method is also studied. The numerical and experimental results demonstrate the efficiency of the proposed method for crack detection. It is shown that SWT of the modal data of cracked simply-supported beams provides a better crack indication than conventional DWT. The relationship between SWT detail coefficient and crack size (depth and width) are also discussed. A new method based


on the average difference of the SWT detail coefficient of vibration bending modes of a cracked beam and an intact beam is proposed as a damage index and verified.

\section{Keywords}

Small Crack, Crack Detection, Discrete Wavelet Transform, Stationary Wavelet Transform

\section{Introduction}

A crack is a damage that often occurs in structural members and may cause serious failure of structures. It is well known that crack effects are more noticeable in the response of vibrating cracked structures when the crack depth is significant in comparison to the depth of the structure. But if the crack is relatively small, it is difficult to detect. For structural safety, a crack must be detected in the early state. However, in some cases of the structures with external covering and insulation, it is difficult to recognize most cracks by using visual inspection techniques; they may be detected by non-destructive techniques (NDT). Inspection of the structural components for damage is important for making decision on the maintenance program of the structure. System identification is an important tool in the dynamic identification for such purpose. It has gained increasing attention from the scientific community and there has been a lot of research in the last two decades. As a result, a variety of analytical, numerical and experimental investigations now exist. Any crack or localized damage in a structure reduces the stiffness and increases the damping in the structure. Reduction in stiffness is associated with decreases in the natural frequencies and modification of the mode shape of the structure. Many researchers have used one or more of the above characteristics to detect and locate a crack.

Adams et al. [1] described a method of non-destructively evaluating the integrity of structures and applied it to structures. It was shown how vibration measurements made at a single station in the structure can be used, in conjunction with a suitable theoretical, to indicate both the location and the magnitude of a defect. Narkis [2] indicated that the data on the variation of the first two natural frequencies is sufficient for identification of the crack location of a cracked 
simply supported uniform beam. On the basis of changes in the natural frequencies, Messina et al. [3,4] calculated the Damage Location Assurance Criterion, which was used to identify single defect and later extended to identify multiple damage sites. Salawu [5] carried out a review of research work on crack detection based on the change in natural frequencies. Changes in measured dynamic flexibility in structures are also used in damage detection. Zhong et al. [6] proposed a response-only method for structural damage detection using the corrected natural frequency curve of a damaged beam with a traversing auxiliary mass. Pandey et al. [7] evaluated the changes in the flexibility matrix of a structure to identify the presence of damage and locate the damage. Doebling et al. [8] provided an overview of methods to detect, locate, and characterize damage in structural and mechanical systems by examining changes in measured vibration response. Yang et al.[9] used the eigenparameter decomposition of structural flexibility change in structural damage detection. Barone et al. [10] the phase of structural response signal in damage detection in the structure with a low damage which results in a low stiffness variation. Frizzarin et al. [11] developed a baseline-free, time-domain damage detection method based on analysis of nonlinear damping from measured structural vibration responses.

Several researchers have used mode shape measurements to detect damage. Pandey et al. [12] showed that absolute changes in the curvature mode shapes are localized in the region of damage and hence can be used to detect damage in a structure. The change in the curvature mode shapes increase with increasing size of damage. This information can be used to obtain the amount of damage in the structure. Ratcliffe [13] found that the mode shapes associated with higher natural frequencies can be used to verify the location of damage, but they are not as sensitive as the lower modes. Modal curvatures seem to be locally much more sensitive to damage than modal displacements. Abdel [14] investigated the application of the change in modal curvatures to detect damage in a pre-stressed concrete bridge, and also a damage indicator called `curvature damage factor' was introduced.

The accuracy of locating the damage directly depends on the sampling interval. Lower spatial sampling interval provides higher accurate crack location. Modern instrumentation, such as Laser Doppler Vibrometers (LDV), allows for high density of sampling and high precision of 
mode shape measurements. Many publications have been focused on mode shape measurements based on scanning laser. Stanbridge et al. [15] used a Continuous-Scan Laser Doppler Vibrometers (CSLDV) to give the response mode shape of a vibrating surface as a spatial polynomial series. Second spatial derivative of the deflection equation were derived and then stresses and strains were obtained from the curvature equation. Also, the paper presented a method to obtain accurate stress and strain distributions using only five terms in the mode-shape polynomial series. Ho and Ewins [16] presented an analysis technique that uses only response data from CSLDV to determine both the mode shape modulus and the temporal phase relationships of a structure by means of the Time Domain Fourier Filter Output (TDFFO) method. The technique does not need any reference force signal.

Damage detection using wavelet transform is a recent area of research in structural and machine health monitoring. The main advantage gained by using wavelets is the ability to perform local analysis of a signal, i.e., to zoom in on any interval of time or space. Wavelet analysis is thus capable of revealing some hidden aspects of the data that other signal analysis techniques fail to detect. This property is particularly important for damage detection applications. Staszewski et al. [17-18] presented an application of the continuous wavelet transform (CWT) in machinery diagnostics. Surace et al. [19] used the CWT for detecting structural damage in mechanical systems. A review is provided by Peng at al. [20] of available wavelet transformation methods and their application to machine condition monitoring. Douka et al. [21] analyzed the fundamental vibration mode of a cracked cantilever beam using CWT to estimate the location and size of the crack. Gentile et al. [22] used the CWT to detect the location of open cracks in damaged beams by minimizing measurement data and baseline information of the structure. Kim et al. [23] employed the CWT of the measured signal of bending wave in a beam and performed the ridge analysis in order to extract the magnitudes of the incident and the reflected waves for a range of frequencies of interest from the measured wave signal. Rucka et al. [24] applied CWT in estimation of the damage location in beam and plate structures. Spanos et al. [25] proposed CWT for damage detection in Euler-Bernoulli beams by using the difference between the displacement responses of the damaged and the undamaged beams for various 
loading conditions. Bayissa et al. [26] presented a new damage identification technique based on the statistical moments of the energy density function of the vibration responses in the time-scale (or time-frequency) domain. The CWT is conducted to decompose the vibration responses into discrete energy distribution as a joint function of time and scale. An analytical approach is developed by Li et al. [27] for seismic ground motions by applying the CWT for detection and assessment of progressive damage in structural system.

Lu et al. [28] presented a wavelet transform-based method for the detection of structural damage, by comparison of the DWT of the signals before and after damage in the spatial domain. Liew et al. [29] found that crack location could be indicated by the variation of some wavelet coefficients along the length of a structural component. Furthermore, Deng [30] applied directly the DWT to structural response signals to locate a crack along the length of a beam. Al-khalidy et al. [31] applied the orthonormal DWT to the detection of fatigue signals from the observed signals. Hou et al. [32] provided numerical simulation data from a simple structural model with breakage springs and from the DWT of the response curve showed clear spikes, which were attributed to the occurrence of structural damage. In conclusions, the authors state that structural damage or the change in system stiffness may be detected by spikes in details of the wavelet decompositions of the response data. Reda Taha et al. [33] presented a utilitarian view of wavelet transforms (CWT and DWT) and their technologies for structural health monitoring. Smith et al. [34] applied DWT vibration detection in aircraft health monitoring. Grabowska et al. [35] used DWT with propagating Lamb waves for identification of the fatigue crack. Beskhyroun et al. [36] applied DWT for structural damage detection and health monitoring. The method examined characteristics of representative vibration signals under DWT for damage identification.

Though DWT is widely used in the field of structural and machine health monitoring, it is a non-redundant decomposition analysis [37]. The drawback of non-redundant transform is their noninvariance in time/space, i.e., the coefficients of a delayed signal are not a time-shifted version of those of the original signal. Time invariance is very important for feature detection, 
while DWT algorithm does not meet that requirement [38]. SWT was introduced to make the wavelet decomposition time invariant [38]. This improves the power of wavelet transform in signal de-noising, which is widely used in image processing. Wang et al. [39] applied the SWT method to preprocess the microarray images for removing the random noises. Seddiki et al. [40] have investigated the characteristics of the stationary wavelet transform which provides the texture information in images to source coding with BCH codes for channel correction. SWT doubles the number of input samples at each iteration, which can provide a more accurate estimation of the variances at each level and facilitate the identification of salient features in a signal, especially for recognizing noise or signal rupture. In this way, SWT has a great potential in crack detection.

In this paper, the difference between SWT and DWT algorithm and wavelet de-noising are introduced firstly in section 2 and 3 respectively. Then SWT and DWT are applied on mode shapes of structures in the latter sections 4, 5 and 6 . The proposed damage detection algorithm is that DWT or SWT is used to decompose the mode shapes of structures into approximate coefficients and detailed coefficients. From the detailed coefficients, the damage indications can be obtained when crack is relatively large. When the crack is small and the noise is significant, the wavelet de-noising is employed to improve the detailed coefficients and to give more clear damage indication. The comparison results of DWT and SWT in section 5.4 demonstrate that SWT gives better damage indication than DWT in structural damage detection. In the present work, both simulated and experimental results show that the proposed method, SWT of modal data, has great potential in crack detection of beam-like structures.

For simulated model, the damaged simply supported are studied using the ABAQUS finite element method (FEM). The mode shape of damage beams is decomposed by SWT or DWT into a smooth curve, called approximation coefficient, and detail coefficient which includes crack information that is useful for damage detection, respectively. Therefore, a crack in the simply supported beam can be detected by detail coefficient of SWT or DWT decompositions of the modal data obtained from theoretical finite element computations. However, for real cases, 
mode shape is affected by experimental noise. Therefore, the effect of noise on the proposed method is also studied. When the crack is relatively small and noise is significant, the DWT decomposition detail coefficients either performs poorly or not all. The results show the efficiency of the proposed method for crack detection, and they also show that SWT decomposition provides better crack indication than conventional DWT. Thus, the advantages of using SWT rather than DWT of modal data are demonstrated.

To verify the efficiency and practicability of the proposed method, thirty six cases with cracks of varying depths and widths using different spatial sampling intervals and in the presence of up to $10 \%$ random noise are studied in the present work. All the modal displacement data are obtained at different sampling distances (which correspond to number and locations of sensors in experimental tests). The results, for the first four mode shape, show that all the cases can provide evidence of crack existence at the correct location of the beam. Also, the relationships between SWT decomposition detail coefficient and crack size (depth and width) are also discussed. Then a new damage indicator or index is proposed for real applications. This is based on the average difference between the SWT decomposition detail coefficients of cracked and intact beams, which give better crack indicator for real crack detection in beams. Finally, the advantages and disadvantages of the proposed method are also discussed.

\section{Stationary versus discrete wavelet transforms}

Both stationary and discrete wavelets are derived from analytical functions called scaling functions $\phi$ which have two key properties (KP1 and KP2), namely:

KP1: The analytical scaling function $\phi(x)$ and all its integer translations $\phi(x+i)$ form an orthonormal set in $L^{2}$, so that [41]

$$
\int_{-\infty}^{+\infty} \phi(x)^{2} d x=1 \text { and } \int_{-\infty}^{+\infty} \phi(x) \phi(x+i) d x=0
$$


for integers $i \neq 0$.

KP2: The analytical scaling function $\phi(x)$ satisfies the so called two-scale equation [38]:

$$
2^{-1 / 2} \phi\left(\frac{x}{2}-k\right)=\sum_{n=-\infty}^{+\infty} h(n-2 k) \phi(x-n)
$$

Similarly, the mother wavelet $\psi(x)$ satisfies the two-scale equation:

$$
2^{-1 / 2} \psi\left(\frac{x}{2}-k\right)=\sum_{n=-\infty}^{+\infty} g(n-2 k) \phi(x-n)
$$

where $\left\{h_{n}\right\}$ and $\left\{g_{n}\right\}$ are, respectively, the impulse responses of low-pass and high-pass paraunitary quadrature mirror filters (QMF's) which obey the mutual orthogonality relation [41] :

$$
\sum_{n=-\infty}^{+\infty} h(n) g(n+2 i)=0
$$

for all integers $i$. At each step $j$, define

$$
\phi_{j, k}(x)=2^{-j / 2} \phi\left(2^{-j} x-k\right)
$$

and

$$
\psi_{j, k}(x)=2^{-j / 2} \psi\left(2^{-j} x-k\right)
$$

The discrete approximation coefficient at resolution $2^{j}$ can be obtained as [38]

$$
c_{j, k} \stackrel{\Delta}{=}\left\langle f(x), \phi_{j, k}(x)\right\rangle \stackrel{\Delta}{=} \int_{-\infty}^{+\infty} 2^{-j / 2} f(x) \phi^{*}\left(2^{j} x-k\right) d x
$$


where * stands for complex conjugation.

The detail coefficients at the resolution $2^{j}$ are obtained as

$$
d_{j, k}=\left\langle f(x), 2^{-j / 2} \psi\left(2^{-j} x-k\right)\right\rangle
$$

According to Eqs. (2) and (7), $c_{j+1, k}$ can be obtained by direct computation from $c_{j, k}$

$$
\begin{aligned}
c_{j+1, k} & =\left\langle f(x), \phi_{j+1, k}(x)\right\rangle \\
& =\left\langle f(x), 2^{-j / 2} \cdot 2^{-1 / 2} \phi\left(\frac{2^{-j} x}{2}-k\right)\right\rangle \\
& =\left\langle f(x), 2^{-j / 2} \sum_{n=-\infty}^{+\infty} h(n-2 k) \phi\left(2^{-j} x-n\right)\right\rangle \\
& =\sum_{n=-\infty}^{+\infty} h(n-2 k)\left\langle f(x), 2^{-j / 2} \phi\left(2^{-j} x-n\right)\right\rangle \\
& =\sum_{n=-\infty}^{+\infty} h(n-2 k) c_{j, n}
\end{aligned}
$$

From Eqs. (3) and (8), similarly, $d_{j+1, k}$ are computed as

$$
d_{j+1, k}=\sum_{n=-\infty}^{+\infty} g(n-2 k) c_{j, n}
$$

Eqs. (9) and (10) are the multi-resolution algorithm of the traditional DWT. But the classical DWT suffers a drawback that it is not a time-invariant transform. This means that, even with periodic signal extension, the DWT of a translated version of the original signal is not, in general, the translated version of the DWT of the original signal. To circumvent this problem, one can resort to a redundant decomposition of the signal as [38],

$$
\tilde{c}_{j, k}=\left\langle f(x), \frac{1}{2^{j / 2}} \phi\left(\frac{x-k}{2^{j}}\right)\right\rangle
$$




$$
\tilde{d}_{j, k}=\left\langle f(x), \frac{1}{2^{j / 2}} \psi\left(\frac{x-k}{2^{j}}\right)\right\rangle
$$

where $\tilde{c}_{j, k}$ and $\tilde{d}_{j, k}$ are, respectively, the discrete approximation coefficient and detail coefficient.

From Eqs. (2) and (11), one can obtain the approximation coefficient of SWT as

$$
\begin{aligned}
\tilde{c}_{j+1, k} & =\left\langle f(x), \frac{1}{2^{(j+1) / 2}} \phi\left(\frac{x-k}{2^{(j+1)}}\right)\right\rangle \\
& =\left\langle f(x), \frac{1}{2^{j}} 2^{-1 / 2} \phi\left(\frac{2^{-j}(x-k)}{2}-0\right)\right\rangle \\
& =\left\langle f(x), \frac{1}{2^{j}} \sum_{l=-\infty}^{+\infty} h(l-0) \phi\left(2^{-j}(x-k)-l\right)\right\rangle \\
& =\left\langle f(x), \frac{1}{2^{j}} \sum_{l=-\infty}^{+\infty} h(l-0) \phi\left(\frac{x-\left(k+2^{j} l\right)}{2^{j}}\right)\right\rangle \\
& =\sum_{l=-\infty}^{+\infty} h(l) \tilde{c_{j, k+2^{j} l}}
\end{aligned}
$$

Similarly, the detail coefficient of SWT can be obtained as

$$
\tilde{d}_{j+1, k}=\sum_{l=-\infty}^{+\infty} h(l) \tilde{d}_{j, k+2^{j} l}
$$

The SWT of original data is not decimated which leads to redundant (overcomplete) representation of the original signal. So it has great potential for feature extraction and facilitates the identification of salient features in a signal.

\section{Wavelet de-noising}

Generally, the wavelet de-noising is achieved via thresholding. The wavelet thresholding procedure removes noise by thresholding only the wavelet coefficient of the detail subbands, while keeping the low resolution coefficients unaltered. There are two thresholding methods frequently used: 
soft-thresholding and hard-thresholding functions. The soft-thresholding rule is normally chosen over hard-thresholding in de-noising.

The hard-thresholding function is defined as

$$
\eta_{h t}= \begin{cases}x, & |x|>t h \\ 0, & |x| \leq t h\end{cases}
$$

where th is the threshold. The general soft-thresholding function is defined by [39]

$$
\eta_{s t}=\operatorname{sgn}(x) \cdot \max (|x|-t h, 0)
$$

The following threshold function th was used in the current work:

$$
\text { th }=\sigma \sqrt{2 \log N}
$$

where $N$ is the signal length and $\sigma$ is the noise standard deviation. Using the soft thresholding, the de-noising procedure in crack detection involves two steps: (i) decompose the signal, and (ii) threshold the detail coefficients to obtain a new coefficient for crack identification. In the two steps, a signal is first decomposed by the wavelet transform (SWT or DWT). Then the decomposition coefficients are thresholded by the soft-thresholding rule. After the thresholding, a new coefficient is obtained which enables crack or damage detection.

Some researchers [21, 22] have investigated the reduction of noise when using CWT. Here, it is worthy to compare their procedure on noise reduction with the wavelet de-noising algorithm employed in the present work. The strategy of noise reduction in ref. [21] is to set a threshold, which is equal to a percentage of the maximum value of the wavelet coefficients and to consider only the coefficients whose absolute values are greater than the threshold. However, there was no discussion on how to obtain the threshold value used. Douka et al. [21] just simply set the threshold value to 0.5 . 
Generally, the applications of CWT on the mode shapes of cracked beams are based on the use of wavelet coefficient obtained by using different scales ([21] and [22]). In addition, Gentile and Messina [22] investigated the use of different analyzing wavelets (i.e. Gaus2, Daub2, Haar etc.) for crack detection. However, the use of DWT or SWT is based on the wavelet decomposition and the detail coefficients can be used for crack detection. This is the major difference between the applications of CWT and DWT or SWT on modal data. In ref. [22], the authors have investigated the relationship between the wavelet scales and the results of noise reduction. They suggested that a tradeoff for wavelet scales is needed when dealing with noisy data using CWT. Therefore, it needs experience and extra time consumption in real applications.

\section{Numerical verification}

\subsection{Evaluation of natural frequencies and mode shapes}

To verify the proposed method, simply supported beams with relatively small cracks are studied using the ABAQUS finite element code. The beams have single-sided transverse cracks with a fixed depth $H_{c}$, a crack width $W_{c}$, and are located at a distance $l_{c}$ from the left support of a beam as shown in Fig.1. The width and depth of the beam are $b$ and $H$, respectively.

The beam models are made of bright mild steel of cross-sectional area $100 \mathrm{~mm} \times 25 \mathrm{~mm}$ with a length of $3000 \mathrm{~mm}$, and the finite element type employed is the 20node 3D brick element which is denoted in the ABAQUS FE package as C3D20R. The material properties of the beams are: Young's modulus $E=210 G P a$, Density $\rho=7850 \mathrm{Kg} / \mathrm{m}^{3}$, the Poisson ratio $v=0.3$. The first 50 natural frequencies and mode shapes of damaged and intact beams are computed using the finite element method. Thirty six cracked beams were consisting of four crack widths $W_{c}=$ 0.1, 0.5, 1.0 and $5.0 \mathrm{~mm}$, three crack depths $H_{c}=1,2$ and $5 \mathrm{~mm}$, and three spatial intervals $x_{S_{i}}^{(r)}=5,25$ and $125 \mathrm{~mm}$. The cracks were located at $500 \mathrm{~mm}$ from the left end of each beam. 
Table 1 shows the natural frequencies and the relative difference of the first four natural frequencies of the intact beam and cracked beams with different crack sizes (depths and widths). It can be seen that the relative difference of natural frequencies between the intact and cracked beams is less than $0.4 \%$ (or $4 \%$ ) when the crack ratio $\left(c_{r}=H_{c} / H\right)$ is up to $20 \%$. Therefore, it will be difficult to use the direct difference of natural frequencies for crack detection in real applications which will be affected by measurement noise such that the accuracy of the frequency measurement will not be as high as shown in Table 1. Therefore, an alternative approach which is based on the mode shapes of the cracked beams is employed in this paper.

The modal approach presented in this paper uses the modal displacement data to identify and locate damage. The approach is sensitive to the spatial sampling intervals used for the modal displacement data. Ho and Ewins [42] have investigated the effect of sampling intervals on damage index. They studied numerically the effect of spatial resolution of the mode shapes on a damage index, which was defined as the ratio of the modal stiffness of the damaged structure to that of the undamaged structure, and which is also the square of the ratio of the mode shape curvatures of the damaged and undamaged structures. They found that the lower the spatial resolution of the mode shapes, the lower was the sensitivity of the damage index to damage. They concluded that spatial resolution plays an important role in reliable damage identification. Therefore the effect of spatial resolution on the damage identification method proposed in this paper is also investigated.

The modal data required to test the sampling interval sensitivity can be acquired by two methods. In the first method, the modal data can be generated using very small finite elements of element size $l_{e}=5 \mathrm{~mm}$. This will enable spatial sampling intervals $\left(x_{S_{i}}\right)$ of $x_{S_{i}}=5,25$ and $125 \mathrm{~mm}$ to be investigated. The use of larger spatial intervals of $25 \mathrm{~mm}$ and $125 \mathrm{~mm}$ than element size $5 \mathrm{~mm}$ will be equivalent to spatial filtering. In the second method, the desired spatial sampling intervals can be used as the element size, i.e. $x_{S_{i}}=l_{e}=5,25$ and $125 \mathrm{~mm}$. In this method, the errors that will be incurred will be due mainly to the numerical errors 
associated with the finite elements due to differing aspect ratios. Therefore, the first investigation was on the effects of element sizes on the predicted modal properties. The modal properties of simply-supported intact beams of length $3 \mathrm{~m}$ were predicted using elements of lengths $l_{e}=5 \mathrm{~mm}, 25 \mathrm{~mm}$ and $125 \mathrm{~mm}$ which correspond to 600, 120 and 24 elements along the length of the beam, respectively. The first four natural frequencies of bending vibration predicted were identical. Similarly, the errors between the mode shapes are negligibly small as can be seen from Fig.2 (a) which shows the difference between the mode shapes predicted using $25 \mathrm{~mm}$ and $5 \mathrm{~mm}$ length elements, and Fig.2 (b) which shows the difference between the mode shapes predicted using $125 \mathrm{~mm}$ and $5 \mathrm{~mm}$ length elements. It is clearly seen from these figures that the maximum difference between the mode shapes predicted using these element size is less than $0.001 \%$. Therefore, elements of lengths 5,25 and $125 \mathrm{~mm}$ can be used with confidence to predict the first four mode shape data of the intact and cracked beams. This will enable the subsequent use of spatial sampling intervals of 5, 25 and $125 \mathrm{~mm}$ for crack identification and location.

Generally, there is no noticeable difference between these first four mode shapes of the intact and damaged beams with small cracks. Hence, it is difficult to detect small cracks in simply-supported beams by the direct comparisons of the mode shape data of the intact and damaged beams. Some other approaches need to be used. The objective of this work is to propose a new approach based on SWT of mode shapes to provide a method for crack detection in beam-like structures with a small crack, whose crack ratio is less than $20 \%$. In this paper, only the wavelet analysis of the first four mode shapes is studied for crack detection of the simply supported beams.

\subsection{Selection of analyzing wavelet}

The mode shapes of a cracked beam with a small crack depth, are apparently single smooth curves. Actually the mode shapes exhibit local peaks or discontinuities in the region of damage which are not visible. The mode shape 'signal' can be approximately considered as that of the 
intact beam contaminated by 'noise' which consists of response noise and the additional response due to the crack. In this way, the signal can be decomposed by wavelet transform (SWT or DWT) into a smooth curve, called approximation coefficient, and detail coefficient which includes crack information that is useful for damage detection as a crack indicator, respectively.

Now, the selection of the analyzing wavelet is left to be discussed, It is well known that the number of vanishing moments is one of the most important factors for the success of wavelets in magnifying local singularities in various applications. Generally, wavelets with higher number of vanishing moments give higher coefficients and more stable performance. However, the effective support of a wavelet will be increased with the number of vanishing moments. Therefore, a tradeoff between number of vanishing moments and adequate localization should be accomplished. After some experimentation a symlet wavelet 'symmetrical 4' having four vanishing moments has been selected and used as analyzing wavelet in the present work.

\subsection{SWT decomposition and wavelet de-noising}

SWT decomposition of the first four mode shapes is firstly studied. Fig.3 (a-1), (b-1), (c-1) and (d-1) show that SWT approximation coefficient of the first, second, third and fourth mode shape 'signal ' of damaged beam with $1 \mathrm{~mm}$ depth, $1 \mathrm{~mm}$ width crack located at $500 \mathrm{~mm}$ from the left

end side of the beam. The modal response data was obtained at spatial intervals $x_{S_{i}}^{(r)}$ of $5 \mathrm{~mm}$, where $r$ is mode number.

As can seen from those figures, all the SWT approximation coefficients are smooth curves, which can not provide any crack information for detection. In Fig.3 (a-2) , (b-2), (c-2) and (d-2), the SWT detail coefficient of the first, second, third and fourth mode shape 'signal', respectively, provide evidence of crack existence at $500 \mathrm{~mm}$ from the left end side of the beam because the detail coefficients exhibit high values at this position. It can be seen that all the detail 
coefficients contain the noise which is due to numerical computational errors associated with the finite element analysis. In this paper, the wavelet soft-thresholding de-noising method is used to remove noise from the SWT decomposition detail coefficients of the first, second, third and fourth mode shape 'signal'; the de-noised detail coefficients are shown in Fig.3 (a-3), (b-3), (c-3) and (d-3). The de-noised results give better crack indicator than those shown in Fig.3 (a-2), (b-2), (c-2) and (d-2).

An important point to be clarified is the difference between the SWT application in the field of image processing and the field of signal processing of modal data discussed in this paper. In the field of image processing, an image signal is first decomposed by wavelet transform, and then the decomposition detail coefficients are thresholded by the thresholding rule to obtain a new detail coefficient. This new de-noised detail coefficient is then reconstructed with approximation coefficient to produce a new image signal, which is a filtered signal by wavelet transform to enhance the image quality [39]. Similar to the steps of image processing, the method proposed in this paper also first decomposes the modal data using wavelet transform, and then the detail coefficients are thresholded by the thresholding rule. After the thresholding, a new coefficient is obtained. This new coefficient contains some information about crack location. It should be noted here that multi-level SWT decomposition is usually needed for image processing, however, in the present work, single-level SWT decomposition is needed in the field of crack detection in beam-like structure. That is, $j$ equals to 1 in the Eqs. (13) and (14). The proposed method using SWT decomposition of modal data has great potential in crack detection in beam-like structures. Further verification of the proposed method will be discussed in the subsequent section.

\section{Further numerical verification of SWT in crack detection}

To verify the efficiency and practicability of the proposed method, the thirty six cases with cracks of varying depths $\left(W_{c}=0.1,0.5,1.0\right.$ and $\left.5.0 \mathrm{~mm}\right)$ and widths $\left(H_{c}=1,2\right.$, and $\left.5 \mathrm{~mm}\right)$

using different spatial intervals $\left(x_{S_{i}}^{(r)}=5,25\right.$, and $125 \mathrm{~mm}$ ) are studied. In this section the 
effects of crack depth, crack width and spatial interval of the mode shape data on the SWT decomposition detail coefficient are investigated.

\subsection{Effects of crack depth}

Fig.4 (a-1), (b-1), (c-1) and (d-1) are, respectively, the SWT decomposition detail coefficients of the first, second, third and fourth mode shapes of cracked beams with $1 \mathrm{~mm}$ width cracks, whose depths are $1 \mathrm{~mm}, 2 \mathrm{~mm}$ and $5 \mathrm{~mm}$. Fig.4 (a-2), (b-2), (c-2) and (d-2) are the zoom of Fig.4 (a-1), (b-1), (c-1) and (d-1) at $500 \mathrm{~mm}$ location, respectively. Both the original and zoomed curves provide evidence of crack existence at $500 \mathrm{~mm}$ from the left end side of the beam because the detail coefficients exhibit high peak values at this position.

In all these three cases, the element length is $5 \mathrm{~mm}$, and the modal displacement data is sampled at $5 \mathrm{~mm}$ spatial intervals $x_{S_{i}}^{(r)}$ along the lengths of the beams resulting in a total of 601 data points. All the modal data are normalized such that the maximum relative amplitude for any mode shape is 1.0. Thus a direct comparison between different cases is possible. As can be seen from Fig.4 (a-2) to (d-2), the SWT decomposition detail coefficients change due to the depth varying from $1 \mathrm{~mm}$ to $5 \mathrm{~mm}$. Comparing the peak values of SWT decomposition detail coefficients in the region of damage, it is seen that the peak value for the case of $H_{c}=1$ is less than for the case of $H_{c}=2$, whereas the peak value for the case of $H_{c}=5$ is the maximum.

It should be noted that all the peak values for these three cases are positive. As the crack depth changes from $1 \mathrm{~mm}$ to $2 \mathrm{~mm}$ and to $5 \mathrm{~mm}$, the peak value of SWT decomposition detail coefficient increases. Also, the peak value of detail coefficient of a higher mode shape is greater than that of a lower mode shape, which can be seen from the original and zoomed curves in Fig.4.

\subsection{Effects of spatial intervals of mode shape data}


When the modal displacement data are sampled at distance interval of $25 \mathrm{~mm}$ along the length of the beams, it results in a total of 121 data points. Fig.5 (a-1), (b-1), (c-1) and (d-1) are, respectively, the detail coefficient of the first, second, third and fourth mode shapes of cracked beams with 1 mm wide cracks, whose depths are 1 mm, 2 mm and 5 mm. Fig.5 (a-2), (b-2), (c-2) and (d-2) are the zoom curves of Fig.5 (a-1), (b-1), (c-1) and (d-1) at $500 \mathrm{~mm}$ location, respectively. The original and zoomed curves provide evidence of crack existence at $500 \mathrm{~mm}$ from the left end of the beam because the detail coefficients exhibit high peak values at this position.

Fig.6 (a-2), (b-2), (c-2) and (d-2), the zoomed curves of Fig.6 (a-1), (b-1), (c-1) and (d-1) show, respectively, the detail coefficients of the first, second, third and fourth mode shape of cracked beams with $1 \mathrm{~mm}$ width crack, whose depths are $1 \mathrm{~mm}, 2 \mathrm{~mm}$ and $5 \mathrm{~mm}$. It can be seen from Fig.6, when sampling distance increases from $5 \mathrm{~mm}$ to $25 \mathrm{~mm}$, the peaks values of SWT decomposition detail coefficients of the first four mode shapes decrease. It decreases at a faster speed when the sampling distance changes from $25 \mathrm{~mm}$ to $125 \mathrm{~mm}$. As a result, all the 'peak' values are negative now; also, the 'peak' value for the case of $H_{c}=5 \mathrm{~mm}$ is less than the peak value for the case of $H_{c}=2 \mathrm{~mm}$, whereas the 'peak' value for the case of $H_{c}=1 \mathrm{~mm}$ is the maximum. For the other cases, similar results can be obtained, namely that the decrease in magnitude of the peak value of SWT decomposition detail coefficient is greater when the spatial interval (sampling distance) between the mode shape data points is a larger value. Also, Fig.5 show that the absolute peak value of the SWT detail coefficient increases as the crack depth increases, and as the mode number increases.

\subsection{Effects of crack width}

Fig.7 (a-1), (b-1) and (c-1) are, respectively, the SWT decomposition detail coefficient of the second mode shape of three cracked beams with cracks of $1 \mathrm{~mm}$ depth, whose widths are $0.1 \mathrm{~mm}, 0.5 \mathrm{~mm}$, $1 \mathrm{~mm}$ and $5 \mathrm{~mm}$. The mode shape data are sampled at $5 \mathrm{~mm}, 25 \mathrm{~mm}$ and $125 \mathrm{~mm}$ interval along the lengths of the beams. Fig.7 (a-2), (b-2) and (c-2) are the zoom curves of Fig.7 (a-1), (b-1) and (c-1) 
at $500 \mathrm{~mm}$ location, respectively. Fig.7 gives an example to show how the peak values of detail coefficient change when crack width and sampling distance have been varied.

Similar to the previous results given, the relation between crack width and the SWT decomposition detail coefficient can be obtained, namely that the decrease in magnitude of the peak value of SWT decomposition detail coefficient is greater when the spatial interval between the mode shape data points is a larger value. Also, Fig.7 show that the absolute peak value of the SWT detail coefficient increases as the crack width decreases, and as the mode number increases. However, it should be noted that the absolute peak values of SWT decomposition detail coefficients do not give a very clear crack indicator for detecting the crack location when the spatial interval is large (i.e. $x_{S_{i}}^{(r)}=125 \mathrm{~mm}$ ). In the section 5.5, a modified approach for real applications using large spatial sampling intervals, is investigated.

\subsection{Comparison of SWT and DWT methods}

In order to compare the efficiency of crack detection based on SWT and DWT of mode shapes 'signal' of damaged beam, SWT and DWT decompositions of the first two mode shapes of a cracked beam with a $0.1 \mathrm{~mm}$ wide crack whose depth is $1 \mathrm{~mm}$, are investigated. Fig.8 (a-1) and (a-2) are the SWT decomposition detail coefficients of the first two mode shapes. As seen from Fig.8 (a-1) and (a-2), the curves provide evidence of crack existence at $500 \mathrm{~mm}$ from the left end of the beam because the detail coefficients exhibit obvious discontinuity at this position. Similarly, Fig.8 (b-1) and (b-2) show the DWT decomposition detail coefficients of the first two mode shapes. But the indication of crack existence at location $l_{c}=500 \mathrm{~mm}$ is not as clear from the DWT curves as is the case with the SWT curves.

In DWT, actually, a down-sampling algorithm is used to perform the transform. That is, one point out of two is kept during transformation. Therefore, the resulting sequences are decimated 
(i.e. only every even member of a sequence is kept), the whole length of sequences will reduce by half after the transformation. This procedure is repeated until the desired level of decomposition is reached. For SWT, the redundant transform, instead of down-sampling, an up-sampling procedure is carried out before performing filter convolution at each level. At first sight, the computational complexity appears to be a drawback of the proposed methods using SWT. However, the computation time of SWT which is less than $40 \mathrm{~ms}$ for spatial measurement points of up to 3001 on a beam of length $3 \mathrm{~m}$, is insignificant when compared to the time of several minutes it will tack to perform the measurements and to carry out the modal analysis. Due to the different procedures between DWT and SWT, the detail coefficient of DWT decomposition has less crack information than that of SWT. Taking an example, the detail coefficients of SWT decomposition of the first two mode shapes shown in Fig.8 (a-1) and (a-2) give a clearer identification and location of the crack, which was located at $l_{c}=500 \mathrm{~mm}$, than the identification provided by DWT decomposition in Fig.8 (b-1) and (b-2). The DWT decomposition does not provide an unambiguous crack identification and location. Therefore, SWT decomposition detail coefficient of mode shape of the cracked beam can provide a better crack indication than conventional DWT.

\subsection{Modified Approach for Real Applications Using Large Spatial Sampling Intervals}

In real applications, except for the use of scanning laser vibrometers ([15] and [16]), it will be difficult to make many measurements along a beam of $3000 \mathrm{~mm}$ length, using a small spatial sampling distance of $5 \mathrm{~mm}$ or $25 \mathrm{~mm}$. The case of sampling distance of $125 \mathrm{~mm}$ will be more reasonable because measurements need to be made only at twenty five points. However,

comparing Fig.6 (for which $x_{S_{i}}^{(r)}=125 \mathrm{~mm}$ ) with Figs. 4 and 5 (for which $x_{S_{i}}^{(r)}=5 \mathrm{~mm}$ and $25 \mathrm{~mm}$ respectively), the peak values of SWT decomposition detail coefficients do not give a very clear crack indicator for detecting the crack location when the spatial interval is large and especially if the crack is small. Therefore, it is proposed that the following equation be used as a damage index for small crack detection in real applications where the number of measurement 
sensors, signal amplifiers or data acquisition channels are limited,

$$
\overline{\Delta d}_{S W T}=\frac{1}{N} \sum_{r=1}^{N} d_{\text {Crack, },}-\frac{1}{N} \sum_{r=1}^{N} d_{\text {Intact }, r}
$$

where $d_{\text {Crack,r }}$ and $d_{\text {Intact, }, \text { }}$ are the SWT detail coefficient of mode shapes of a cracked beam and an intact beam for mode $r, N$ is the number of mode shapes considered, and $\overline{\Delta d}_{S W T}$ is the average difference of the SWT detail coefficient of vibration bending modes between cracked and intact beam. It should be noted that when there is no noise present, there is no need for averaging. The damage index is simply given by

$$
\Delta d=d_{\text {Crack }, r}-d_{\text {Intact }, r}
$$

To test the proposed damage indices, two cases were investigated, namely, ( $\mathrm{i}$ ) when there is no measurement noise (for which Eq. (19) should be used), (ii ) when $10 \%$ measurement noise is present (for which Eq. (19) should be used).

Fig.9 (a), (b), (c) and (d), respectively, show the difference of SWT decomposition detail coefficients of the first four mode shapes of four beams with small cracks of $1 \mathrm{~mm}$ depth and widths $0.1 \mathrm{~mm}, 0.5 \mathrm{~mm}, 1 \mathrm{~mm}$ and $5 \mathrm{~mm}$ and for sampling distance of $125 \mathrm{~mm}$ but without measurement noise. The differences were obtained using Eq. (19). In comparison to Fig.6, the graphs in Fig.9 provide better evidences of crack existence at $500 \mathrm{~mm}$ from the left end of the beam because the global detail coefficient of the intact beam has been subtracted from the global detail coefficient of the cracked beam. The residual response, which is due to the presence of the crack, becomes magnified and, therefore, facilitates the correct identification and location of the crack.

Furthermore, the effect of noise on this approach for applications involving relatively large spatial sampling intervals needs to be verified. This was achieved by adding a random noise of 
magnitude of $10 \%$ of the amplitude of the mode shape data to the mode shape. Fig.10 (a-1) to (d-1) are the SWT detail coefficient of the first four mode shape of a cracked beam ( $H_{c}=1 \mathrm{~mm}$, $\left.W_{c}=1 \mathrm{~mm}\right)$. Fig.10 (a-2) to (d-2) are the corresponding denoised SWT detail coefficient.

The severity of $10 \%$ added noise on the SWT detail coefficient can be appreciated by comparing the case $W_{c}=1 \mathrm{~mm}, H_{c}=1 \mathrm{~mm}, x_{S_{i}}^{(r)}=125 \mathrm{~mm}$ but without added noise in Fig.6, which is shown as dashed lines, with the identical case but with 10\% added noise shown in Fig.10. It is seen that the fairly smooth and regular identifiable shapes observed for four modes in Fig.6 become distorted, irregular and unidentifiable shapes in Fig.10. Consequently, it is not possible to use the noisy SWT detail coefficients shown in Fig.11 for crack identification and location. However, the effect of noise can be minimised by averaging and differencing using Eq. (18) to enhance the crack information.

Fig.11 (a-1) shows the average of the SWT detail coefficient of the first four mode shape of the cracked beam. Fig.11 (a-2) is the denoised average of the SWT detail coefficient. Though the crack information is magnified by averaging the SWT detail coefficient of vibration bending modes, it is still hard to carry out crack detection. Similarly, Fig.11 (b-1) and (b-2) show the averaged SWT detail coefficient, and the denoised average of the SWT detail coefficient of the first four modes of the intact beam whose mode shape data was also subjected to $10 \%$ random noise. It should be noted that it has been assumed that both the intact and cracked beams are assumed to be tested under the same conditions so that their response is subject to the same measurement noise. Fig.11 (c-1), (c-2) are, respectively, the average difference of SWT detail coefficients between cracked and intact beams, and the denoised result. These two figures show that the effects of the $10 \%$ added noise are further reduced. Consequently, the effects of the crack on the response are further enhanced. Therefore, it is now possible to clearly identify and locate the crack as the figures now provide clear and unambiguous evidence of crack existence at $500 \mathrm{~mm}$ from the left end of the beam. 


\section{Experimental verification of SWT in crack detection}

Experimental tests using a simply-supported aluminum beam were conducted. The dimensions of the damaged beam are $L \times H \times B=2400 \times 25 \times 100 \mathrm{~mm}^{3}$. A crack, whose depth is $2.5 \mathrm{~mm}$, was located at $l_{c}=0.4 \mathrm{~m}$. Fig. 12 shows the experimental set-up used for testing. A random signal was generated and then amplified by a power amplifier, and exerted on the beam structure through a shaker. The response signal and input signal were respectively sensed by a PCB accelerometer and a PCB force sensor. The displacement data is sampled at $100 \mathrm{~mm}\left(x_{s}=100 \mathrm{~mm}\right)$ interval along the lengths of the beam resulting in a total of 25 data points.

In order to compare the efficiency of crack detection based on SWT and DWT of mode shape of this damaged beam, SWT and DWT decompositions of the first mode shape are investigated. Fig.13 (a-1) and (a-2) are the DWT decomposition detail coefficients of the first mode shape. As seen from Fig.13 (a-1) and (a-2), the curves can not provide evidence of crack existence at 400 $\mathrm{mm}$ from the left end of the beam. Similarly, Fig.13 (b-1) and (b-2) show the SWT decomposition detail coefficients of the first mode shape. The curves provide obvious evidence of crack existence at $400 \mathrm{~mm}$ from the left end of the beam because the detail coefficients exhibit obvious discontinuity at this position. The results show that SWT of the modal data of cracked simply-supported beams provides a better crack indication than conventional DWT. This reason is that the detail coefficient of DWT decomposition has less crack information than that of SWT.

\section{Concluding remarks}

This paper proposes a new approach based on SWT to provide a method for small crack detection in beam-like structures. The modal responses of the damaged simply supported beams used are computed using the finite element method. Crack identification using the SWT and the DWT of the first four mode shapes has been compared. In real applications, the mode shape 
data is affected by experimental noise. Therefore, the effects of a normally distributed random noise have also been studied. The numerical and experimental results demonstrate the efficiency of the proposed method for crack detection. Though crack information can be obtained from the detail coefficient of the SWT or the DWT of mode shapes, DWT is a down-sampling algorithm whereas SWT is an up-sampling one. This is the main difference between SWT and DWT, and the fundamental reason why SWT of mode shape provides better crack identification than conventional DWT, especially, when the crack is relatively small and noise is significant. Also, SWT decomposition has better anti-noise ability than DWT decomposition. Therefore, SWT of modal data has great potential in crack detection of beam-like structures.

\section{Nomenclature}

$\begin{array}{ll}\phi & \text { scaling function } \\ \psi & \text { mother wavelet } \\ h_{n} & \text { the impulse responses of low-pass paraunitary quadrature mirror filter } \\ g_{n} & \text { the impulse responses of high-pass paraunitary quadrature mirror filter } \\ c_{j, k} & \text { approximation coefficient of DWT decomposition } \\ d_{j, k} & \text { detail coefficient of DWT decomposition } \\ \tilde{c}_{j, k} & \text { approximation coefficient of SWT decomposition } \\ \tilde{d}_{j, k} & \text { detail coefficient of SWT decomposition }\end{array}$




\begin{tabular}{|c|c|}
\hline$\eta_{h t}$ & hard-thresholding function \\
\hline$\eta_{s t}$ & soft-thresholding function \\
\hline th & threshold \\
\hline$N$ & signal length \\
\hline$\sigma$ & noise standard deviation \\
\hline$l_{c}$ & crack location of cracked beam from the left support \\
\hline$l$ & length of beam \\
\hline$l_{e}$ & element size \\
\hline$b$ & width of beam \\
\hline$H$ & depth of beam \\
\hline$H_{c}$ & crack depth \\
\hline$W_{c}$ & crack width \\
\hline E & Young's modulus of material \\
\hline$\rho$ & density of material \\
\hline$v$ & Poisson ratio of material \\
\hline
\end{tabular}




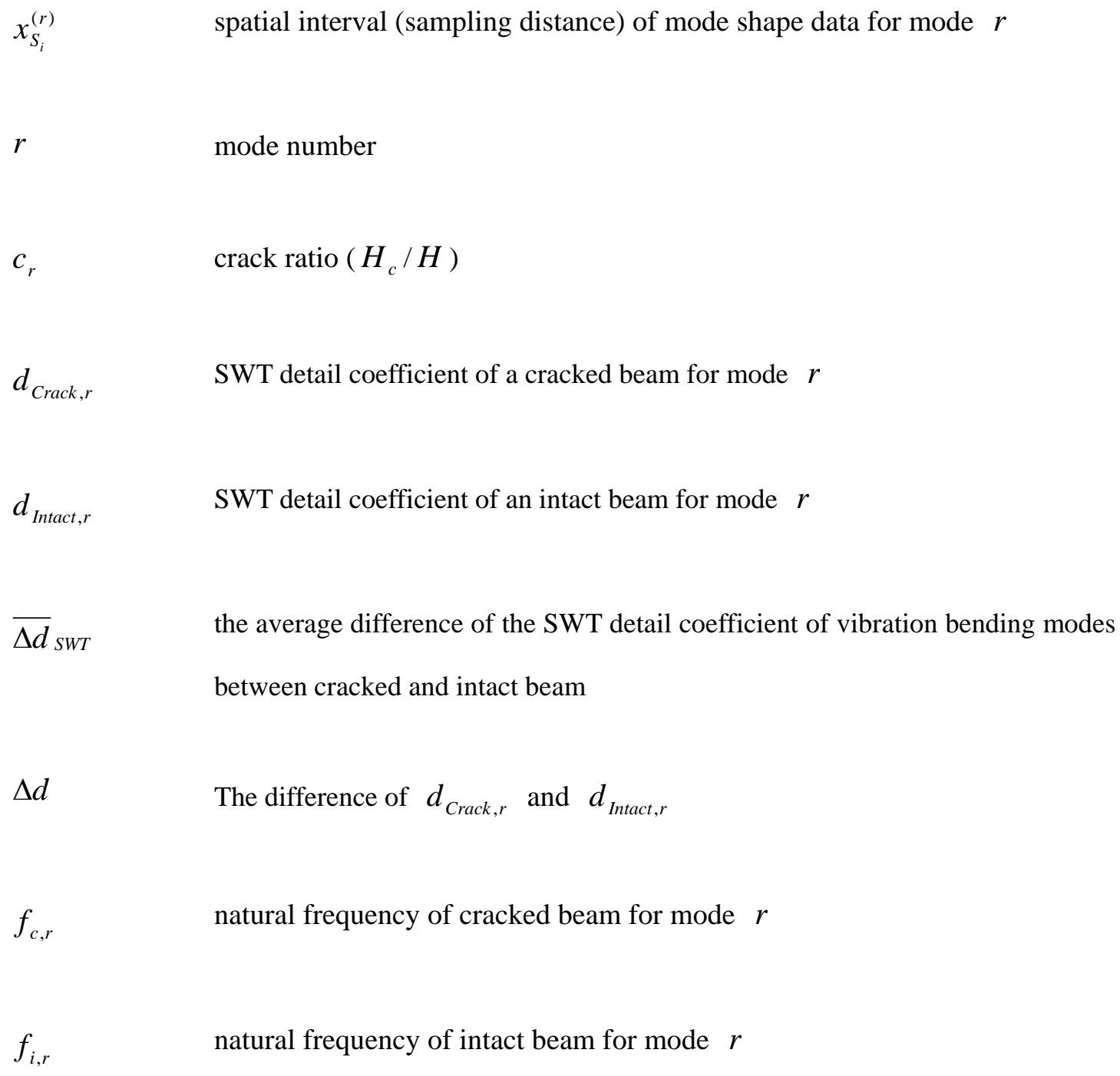

\section{References}

1. Adams R.D., Cawley P., Pye C.J., Stone B.J., A vibration technique for non-destructively assessing the integrity of structures, Journal of Mechanical Engineering Science 1978; 20: 93-100

2. Narkis, Y., Identification of crack location in vibrating simply supported beams, Journal of Sound and Vibration 1994; 172: 549-558.

3. Messina, A., Jones I.A. Damage detection and localization using natural frequency change, 14th International Modal Analysis Conference, Orlando, 1996, 67-76. 
4 Messina, A., Williams E.J., Contursi T. Structural damage detection by sensitivity and statistical-based method, Journal of Sound and Vibration 1998; 216(5): 791-808.

5. Salawu, O. S., Detection of structural damage through changes in frequencies: a review, Engineering Structures 1997; 19: 718-723

6. Zhong, S., Oyadiji, S.O., Ding K., Response-only method for damage detection of beam-like structures using high accuracy frequencies with auxiliary mass spatial probing, Journal of Sound and Vibration 2008; 311: 1075-1090

7. Pandey, A. K. and Biswas M., Damage detection in structures using change in flexibility, Journal of Sound and Vibration 1994; 169: 3-17

8. Doebling S.W., Farrar C.R., Prime M.B., A summary review of vibration-based damage identification methods, The Shock and Vibration Digest 1998; 30(2): 91-105

9. Yang Q.W., Liu J.K., Damage identification by the eigenparameter decomposition of structural flexibility change, International Journal for Numerical Methods in Engineering 2008.

10. Barone G., Marino F., Pirrotta A., Low stiffness variation in structural systems: Identification and localization, Structural Control and Health Monitoring 2008; 15: 450-470

11. Frizzarin M., Feng M. Q., Franchetti P., Soyoz S., Modena C., Damage detection based on damping analysis of ambient vibration data, Structural Control and Health Monitoring 2008.

12. Pandey, A.K., Biswas, M., Samman, M.M., Damage detection from changes in curvature mode shapes, Journal of Sound and Vibration 1991; 145 (2): 321-332

13. Ratcliffe, C. P., Damage detection using a modified Laplacian operator on mode shape data, Journal of Sound and Vibration 1997; 204: 505-517

14. Abdel, W.M.M., Roeck, G. De, Damage detection in bridges using modal curvatures: application to a real damage scenario, Journal of Sound and Vibration 1999; 226(2): 217-235

15. Stanbridge, A. B., Martarelli, M., Ewins, D. J., Measuring strain response mode shapes with a continuous-scan LDV, Proceedings of SPIE - The International Society for Optical Engineering 2000; 4072: 160-168 
16. Ho, Y. K. and Ewins, D. J., Reference-free mode shape identification with a continuously scanning laser Doppler vibrometer (SLDV), Proceedings of SPIE - The International Society for Optical Engineering 2001; 4317: 604-609.

17. Staszewski W.J., Tomlinson G.R., Application of the wavelet transform to fault detection in a spur gear, Mechanical Systems and Signal Processing 1994; 8(3): 289-307

18. Staszewski W.J., Structural and mechanical damage detection using wavelets, The Shock and Vibration Digest 1998; 30(6):457-72.

19. Surace C., Ruotolo R., Crack detection of a beam using the wavelet transform, Proceedings of the 12th International Modal Analysis Conference 1994, Honolulu, Hawaii: 1141-1167

20. Peng, Z.K., Chu, F.L., Application of the wavelet transform in machine condition monitoring and fault diagnostics: a review with bibliography, Mechanical Systems and Signal Processing 2004; 18: 199-221.

21. Douka, E., Loutridis, S., Trochidis, A., Crack identification in beams using wavelet analysis, International Journal of Solids and Structures 2003; 40: 3557-3569.

22. Gentile, A., Messina, A.,On the continuous wavelet transforms applied to discrete vibrational data for detecting open cracks in damaged beams, International Journal of Solids and Structures 2003; 40: 295-315.

23. Kim I.K., Kim Y.Y., Damage size estimation by the continuous wavelet ridge analysis of dispersive bending waves in a beam, Journal of Sound and Vibration 2005; 287: 707-722.

24. Rucka M., Wilde K., Application of continuous wavelet transform in vibration based damage detection method for beams and plates, Journal of Sound and Vibration 2006; 297: 536-550.

25. Spanos P., Failla G., Santini A., Pappatico M., Damage detection in Euler-Bernoulli beams via spatial wavelet analysis, Structural Control and Health Monitoring 2006; 13 472-487.

26. Bayissa W.L., Vibration-based structural damage identification using wavelet transforms, Mechanical Systems and Signal Processing 2008; 22: 1194-1215. 
27. Li H., Yi T., Gu M., Huo L., Evaluation of earthquake-induced structural damages by wavelet transform, Progress in Natrual Science 2009; 19: 461-470.28. Lu, J., Hsu, Y.T., Vibration analysis of an inhomogeneous string for damage detection by wavelet transform, International Journal of Mechanical Sciences 2002; 44: 745-754.

29. Liew, K.M., Wang, Q., Application of wavelet theory for crack identification in structures, Journal of Engineering Mechanics 1998; 124: 152-157

30. Deng, X. Wang, Q., Crack detection using spatial measurements and wavelet, International Journal of Fracture 1998; 91: 23-28

31. Al-khalidy A., Noori M., Hou Z., Carmona R., Yamamoto S., Masuda A., A study of health monitoring systems of linear structures using wavelet analysis. ASME PVP 1997; 347: 49-58

32. Hou Z., Noori M., Amand R., Wavelet-based approach for structural damage detection, J Engng Mech ASCE 2000; 126(7): 677-83

33. Reda Taha M.M., Noureldin A., Lucero J.L., Baca T.J., Wavelet transform for structural health monitoring: A compendium of of uses and features, Structural Health Monitoring 2006; 5(3): 267-295

34. Smith C., Akujuobi C.M., Hamory P., Kloesel K., An approach to vibration analysis using wavelets in an application of aircraft health monitoring, Mechanical Systems and Signal Processing 2007; 21: 1255-1272.

35 Grabowska J., Palacz M., Krawczuk M., Damage identification by wavelet analysis, Mechanical Systems and Signal Processing 2008; 22: 1623-1635.

36. Beskhyroun S., Oshima T., Mikami S., Wavelet-based technique for structural damage detection, Structural Control and Health Monitoring 2009.37. Mallat S., A theory for multiresolution signal decomposition: The wavelet representation, IEEE Trans. Pattern Anal. Machine Intell.1989; 11: 674-693.

38. Pesquet, J. C., Krim, H., and Carfantan, H., Time-invariant orthonormal wavelet representations, IEEE Trans. Signal Processing 1996; 44: 1964-1970. 
39. Wang, X.H., Istepanian, R. S. H. and Song, Y.H., Microarray Image Enhancement by Denoising Using Stationary Wavelet Transform, IEEE transactions on Nanobioscience 2003; 2 (4): 184-189

40. Seddiki, A., Djebbari, A., Rouvaen, J.M., Using stationary wavelet transform in BCH image coding, Technical Acoustics Electronic Journal 2003 (1)

41. Nason, G.P. and Silverman, B.W., The stationary wavelet translation and some statistical applications, in: A.ANTONIADIS and G.OPPENHEIM (eds.), wavelet and statistics 1995; 281 299, Lecture Notes in Statistics, Springer Verlag.

42. Ho, Y. K. and Ewins, D. J., Numerical evaluation of the damage index, Proceedings of the $2^{\text {nd }}$ International Workshop on Structural Health Monitoring, Stanford University, 1999; 995 1011. 
Table 1: Comparison of first four natural frequencies of intact beam and cracked beams with different crack depths and widths (25mm long elements used).

\begin{tabular}{|c|c|c|c|c|c|c|c|c|c|}
\hline \multirow{2}{*}{\multicolumn{2}{|c|}{ Tases }} & \multicolumn{4}{|c|}{ Natural Frequencies (Hz) } & \multicolumn{4}{|c|}{ Relative Difference * (\%) } \\
\hline & & \multicolumn{4}{|c|}{ Modes } & \multicolumn{4}{|c|}{ Modes } \\
\hline $\begin{array}{c}H_{c} \\
(\mathrm{~mm})\end{array}$ & $\begin{array}{c}W_{c} \\
(\mathrm{~mm})\end{array}$ & 1 & 2 & 3 & 4 & 1 & 2 & 3 & 4 \\
\hline 0 & 0 (intact) & 6.5144 & 26.052 & 58.597 & 104.12 & 0 & 0 & 0 & 0 \\
\hline \multirow[t]{4}{*}{1} & 0.1 & 6.5141 & 26.049 & 58.587 & 104.11 & -0.05 & -0.12 & -0.17 & -0.10 \\
\hline & 0.5 & 6.5141 & 26.048 & 58.586 & 104.11 & -0.05 & -0.15 & -0.19 & -0.10 \\
\hline & 1 & 6.5140 & 26.048 & 58.586 & 104.11 & -0.06 & -0.15 & -0.19 & -0.10 \\
\hline & 5 & 6.5139 & 26.046 & 58.580 & 104.10 & -0.08 & -0.23 & -0.29 & -0.20 \\
\hline \multirow[t]{4}{*}{2} & 0.1 & 6.5133 & 26.039 & 58.557 & 104.07 & -0.17 & -0.50 & -0.68 & -0.48 \\
\hline & 0.5 & 6.5132 & 26.039 & 58.556 & 104.07 & -0.18 & -0.50 & -0.70 & -0.48 \\
\hline & 1 & 6.5132 & 26.038 & 58.555 & 104.07 & -0.18 & -0.54 & -0.72 & -0.48 \\
\hline & 5 & 6.5129 & 26.035 & 58.546 & 104.05 & -0.23 & -0.65 & -0.87 & -0.67 \\
\hline \multirow[t]{4}{*}{5} & 0.1 & 6.5086 & 25.983 & 58.391 & 103.84 & -0.89 & -2.65 & -3.52 & -2.69 \\
\hline & 0.5 & 6.5086 & 25.983 & 58.389 & 103.84 & -0.89 & -2.65 & -3.55 & -2.69 \\
\hline & 1 & 6.5085 & 25.982 & 58.387 & 103.84 & -0.91 & -2.69 & -3.58 & -2.69 \\
\hline & 5 & 6.5077 & 25.972 & 58.358 & 103.80 & -1.03 & -3.07 & -4.09 & -3.07 \\
\hline
\end{tabular}

* Relative Difference $=\frac{f_{c, r}-f_{i, r}}{f_{i, r}} \times 1000 \%$

$f_{c, r}=$ natural frequency of cracked beam for mode $r$

$f_{i, r}=$ natural frequency of intact beam for mode $r$

$\%$ = per thousand 


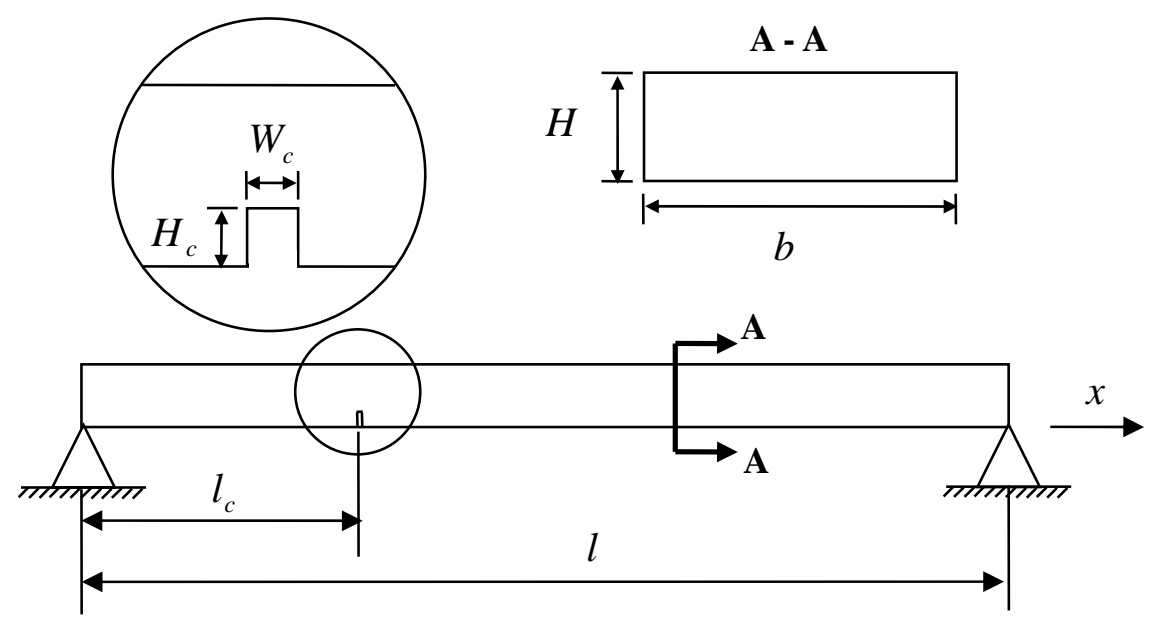

Fig.1. Model of cracked simply supported beam. 

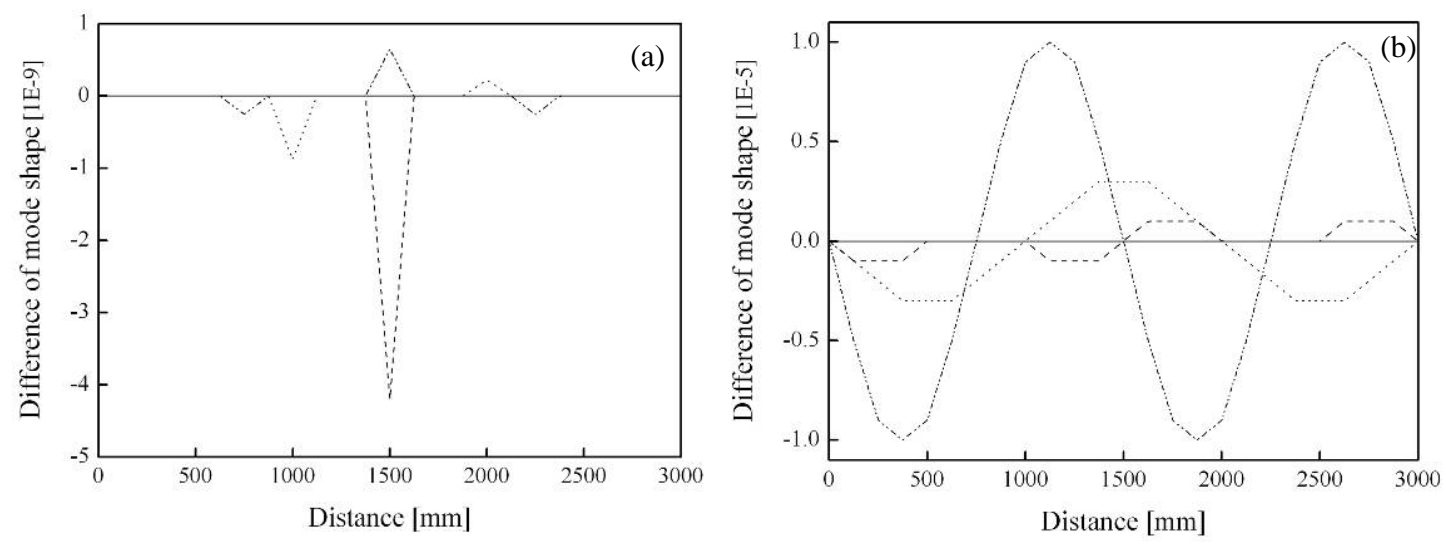

Fig.2. Mode shape difference of an intact beam (3 m length) for different meshes: (a) mode shape difference using $25 \mathrm{~mm}$ and $5 \mathrm{~mm}$ long elements; (b) mode shape difference using $125 \mathrm{~mm}$ and 5 mm long elements. 1st mode, 2nd mode, 3rd mode, -..... 4th mode. 

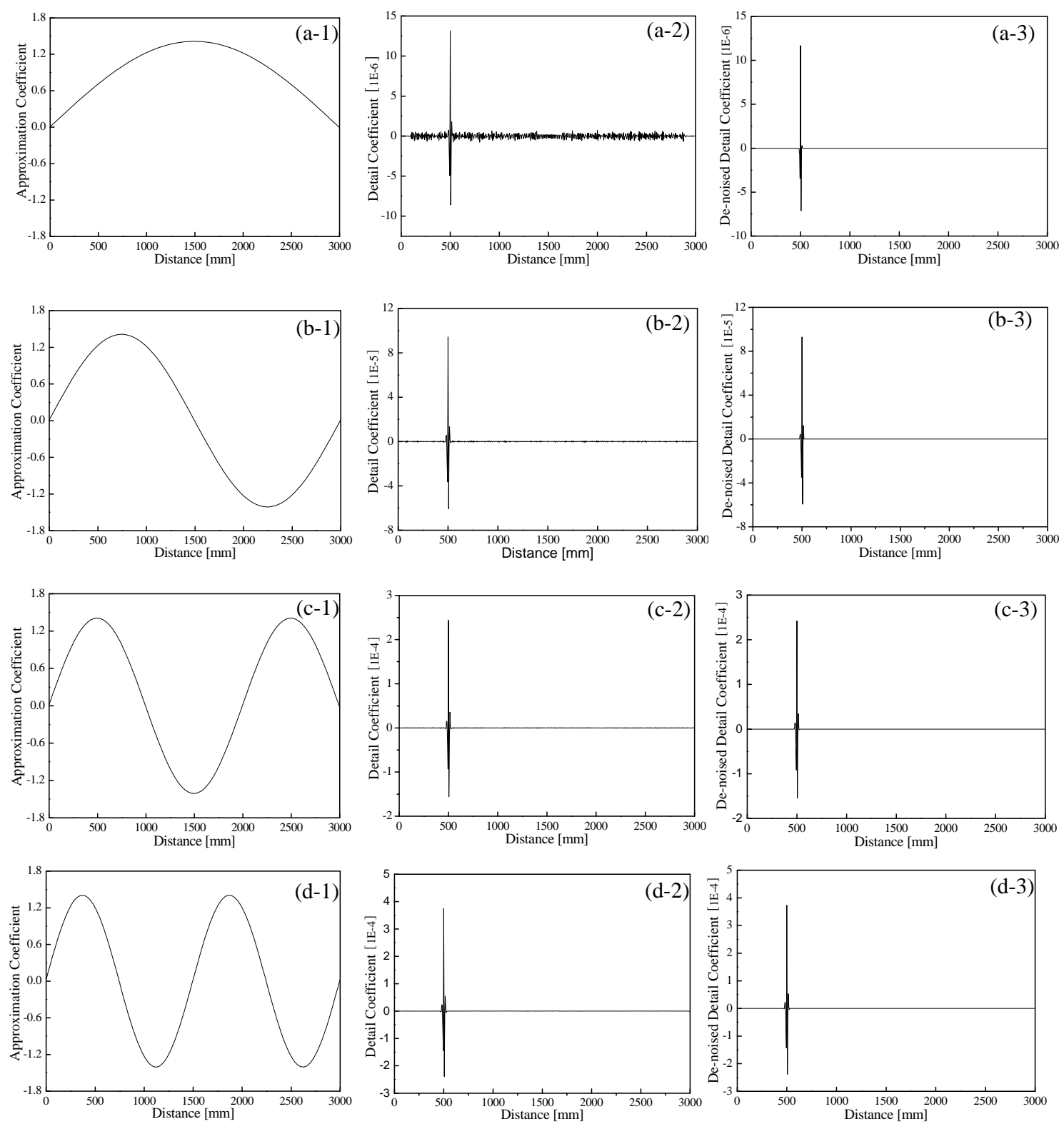

Fig.3. SWT decomposition approximation coefficients (left) and detail coefficients (centre) of the first four mode shapes of a cracked beam $H_{c}=1 \mathrm{~mm}, W_{c}=1 \mathrm{~mm}$, $x_{S_{i}}^{(r)}=5 \mathrm{~mm}$ and wavelet de-noising results (right); (a-1) to (d-1): SWT approximation coefficients for $1^{\text {st }}, 2^{\text {nd }}, 3^{\text {rd }}$ and $4^{\text {th }}$ mode shapes; (a-2) to (d-2): SWT detail coefficients for $1^{\text {st }}, 2^{\text {nd }}, 3^{\text {rd }}$ and $4^{\text {th }}$ mode shapes; (a-3) to (d-3): De-noised SWT detail coefficients for $1^{\text {st }}, 2^{\text {nd }}, 3^{\text {rd }}$ and $4^{\text {th }}$ mode shapes. 

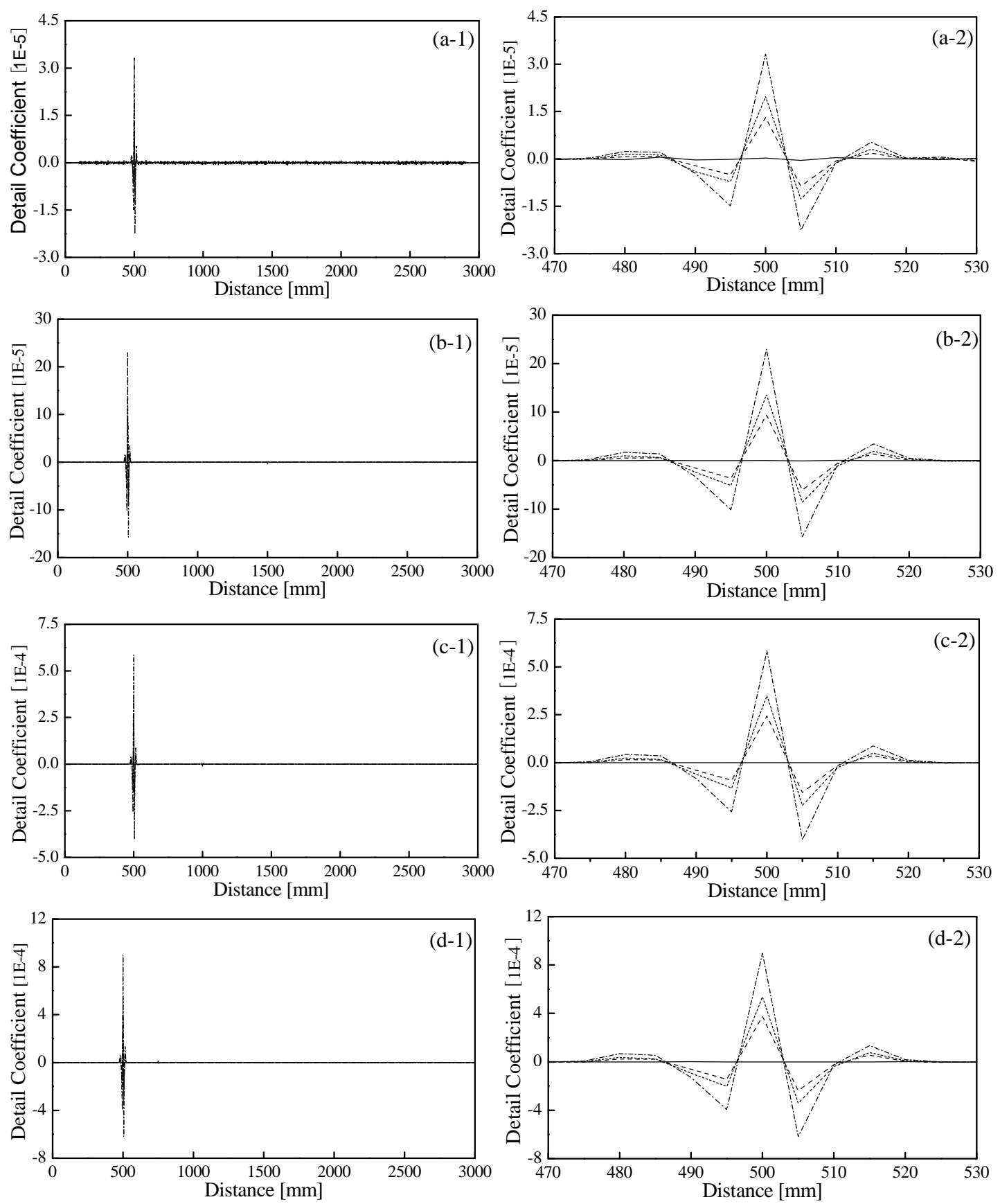

Fig.4. SWT decomposition detail coefficients of cracked beams $W_{c}=1 \mathrm{~mm}, x_{S_{i}}^{(r)}=5 \mathrm{~mm}$ (left) and the zoom curves (right): — Intact, - - - $H_{c}=1 \mathrm{~mm}, \cdots \cdots \cdot-H_{c}=2 \mathrm{~mm}$, -.- $\cdot H_{c}=5 \mathrm{~mm}$; (a-1) to (d-1): SWT detail coefficients of $1^{\text {st }}, 2^{\text {nd }}, 3^{\text {rd }}$ and $4^{\text {th }}$ mode shapes; (a-2) to (d-2): Zoom of SWT detail coefficient of $1^{\text {st }}, 2^{\text {nd }}, 3^{\text {rd }}$ and $4^{\text {th }}$ mode shapes. 

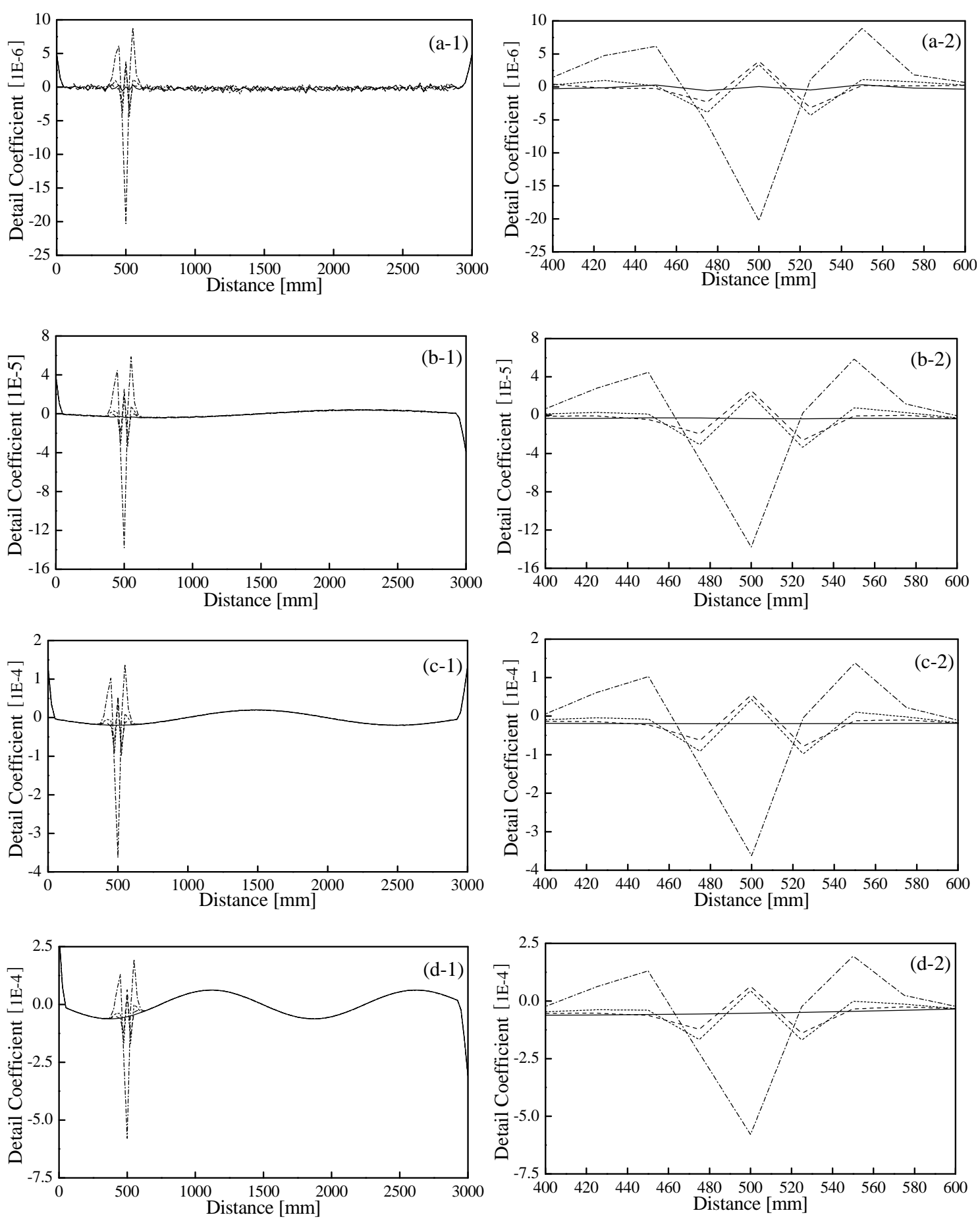

Fig.5. SWT decomposition detail coefficients of cracked beams $W_{c}=1 \mathrm{~mm}, x_{S_{i}}^{(r)}=25 \mathrm{~mm}$ (left) and the zoom curves (right):- Intact, - - - - $H_{c}=1 \mathrm{~mm}, \cdots \cdots \cdot-H_{c}=2 \mathrm{~mm},-\cdot-\cdot \cdot H_{c}=5 \mathrm{~mm}$; (a-1) to (d-1): SWT detail coefficients of $1^{\text {st }}, 2^{\text {nd }}, 3^{\text {rd }}$ and $4^{\text {th }}$ mode shapes; (a-2) to (d-2): Zoom of SWT detail coefficient of $1^{\text {st }}, 2^{\text {nd }}, 3^{\text {rd }}$ and $4^{\text {th }}$ mode shapes. 

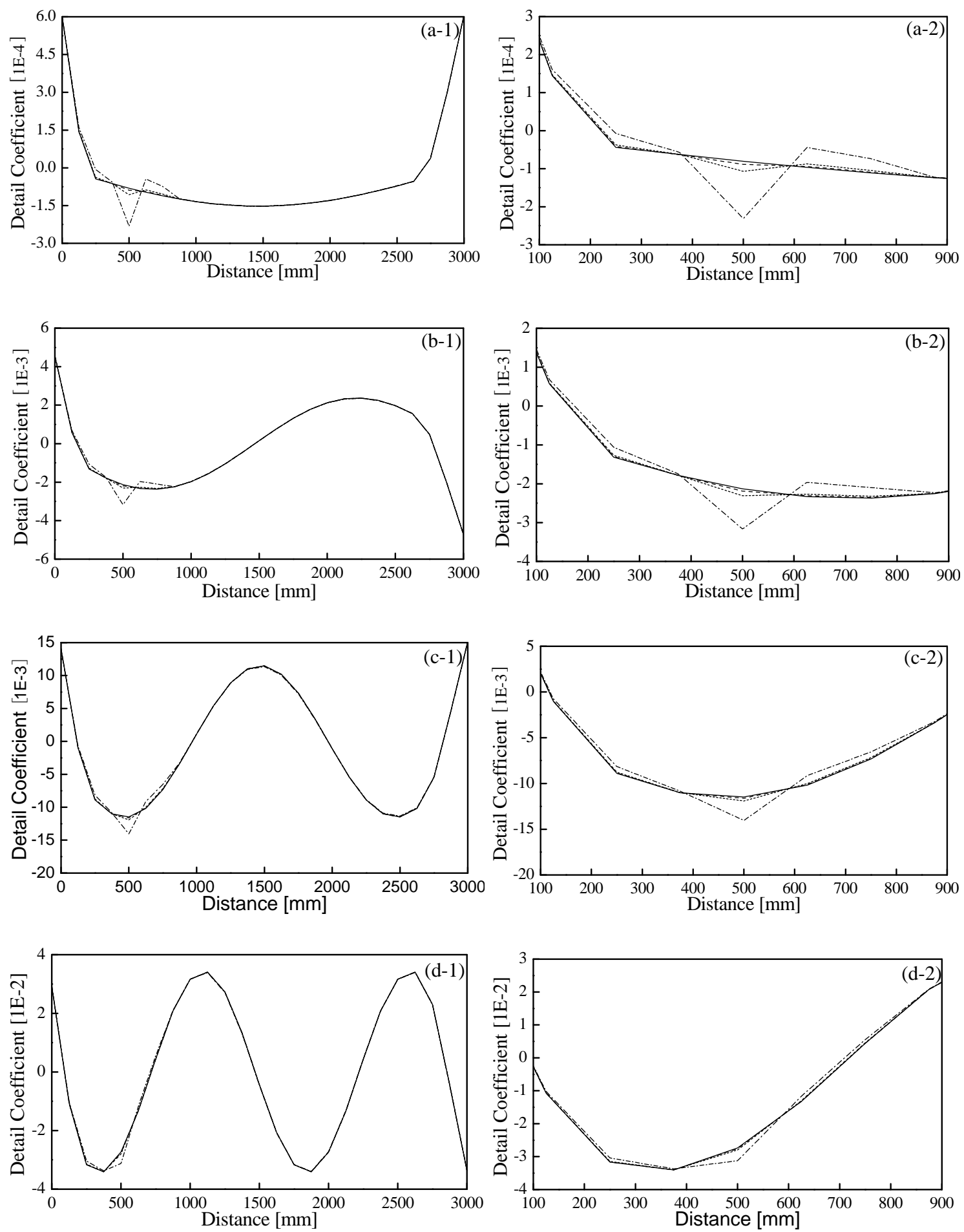

Fig.6. SWT decomposition detail coefficients of cracked beams $W_{c}=1 \mathrm{~mm}, x_{S_{i}}^{(r)}=125 \mathrm{~mm}$ (left) and the zoom curves (right): - Intact, - - - $H_{c}=1 \mathrm{~mm}, \cdots \cdots \cdot-H_{c}=2 \mathrm{~mm}$, $-\cdot \cdot \cdot H_{c}=5 \mathrm{~mm} ;(\mathrm{a}-1)$ to $(\mathrm{d}-1)$ : SWT detail coefficients of $1^{\text {st }}, 2^{\text {nd }}, 3^{\text {rd }}$ and $4^{\text {th }}$ mode shapes; (a-2) to (d-2): Zoom of SWT detail coefficient of $1^{\text {st }}, 2^{\text {nd }}, 3^{\text {rd }}$ and $4^{\text {th }}$ mode shapes. 

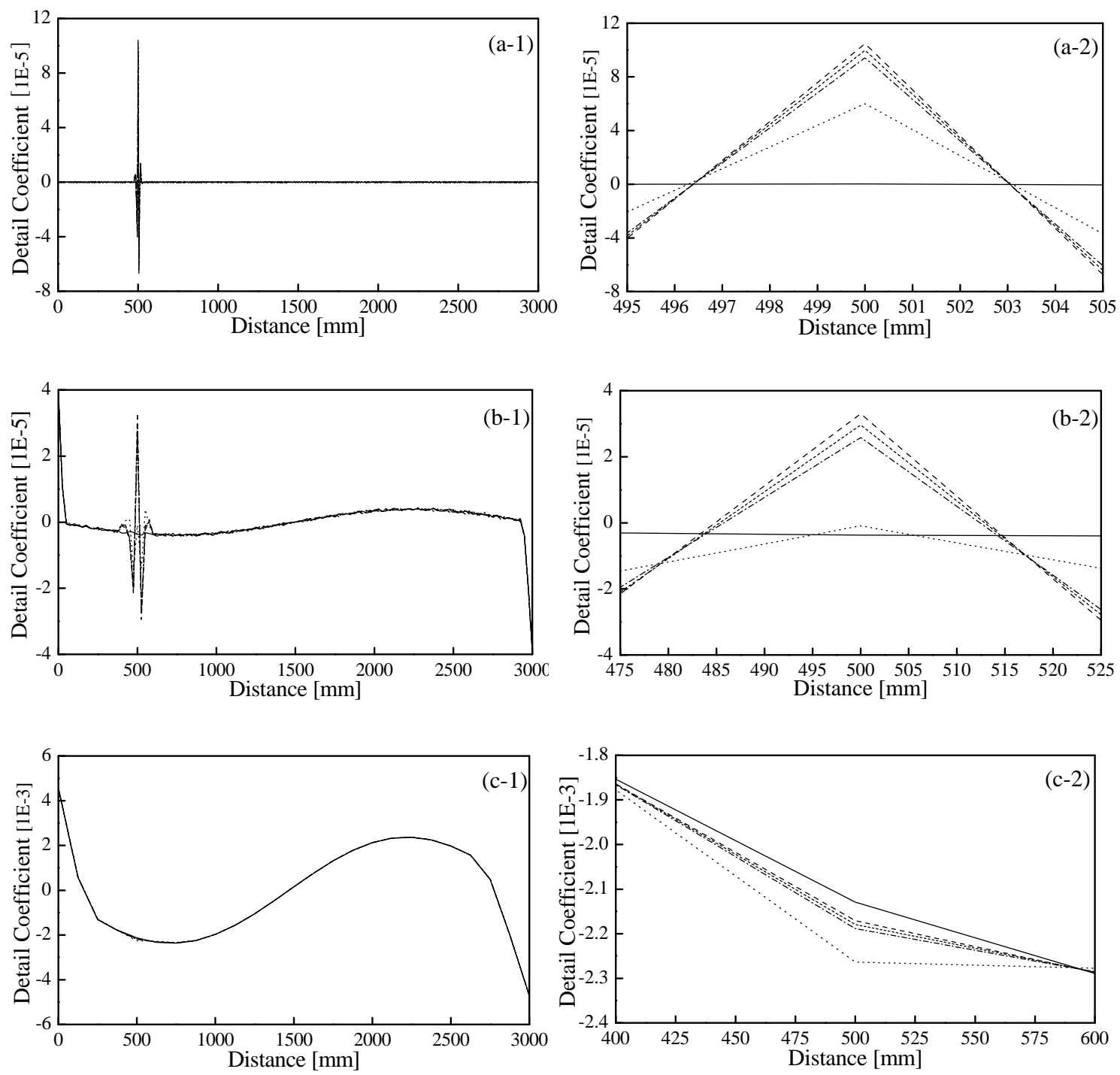

Fig.7. SWT decomposition detail coefficients of the second mode shape of cracked beams $H_{c}=1 \mathrm{~mm}$ (left) and the zoom curves (right) for different spatial intervals: — Intact, - - - $W_{c}=0.1 \mathrm{~mm}, \cdots \cdots \cdot W_{c}=0.5 \mathrm{~mm}, \cdots W_{c}=1 \mathrm{~mm}, \cdots \cdots W_{c}=5 \mathrm{~mm}$. (a-1) SWT detail, $x_{S_{i}}^{(r)}=5 \mathrm{~mm}$; (a-2) Zoom of SWT detail, $x_{S_{i}}^{(r)}=5 \mathrm{~mm}$; (b-1) SWT detail, $x_{S_{i}}^{(r)}=25 \mathrm{~mm}$; (b-2) Zoom of SWT detail, $x_{S_{i}}^{(r)}=25 \mathrm{~mm}$; (c-1) SWT detail, $x_{S_{i}}^{(r)}=125 \mathrm{~mm}$; (c-2) Zoom of SWT detail , $x_{S_{i}}^{(r)}=125 \mathrm{~mm}$. 

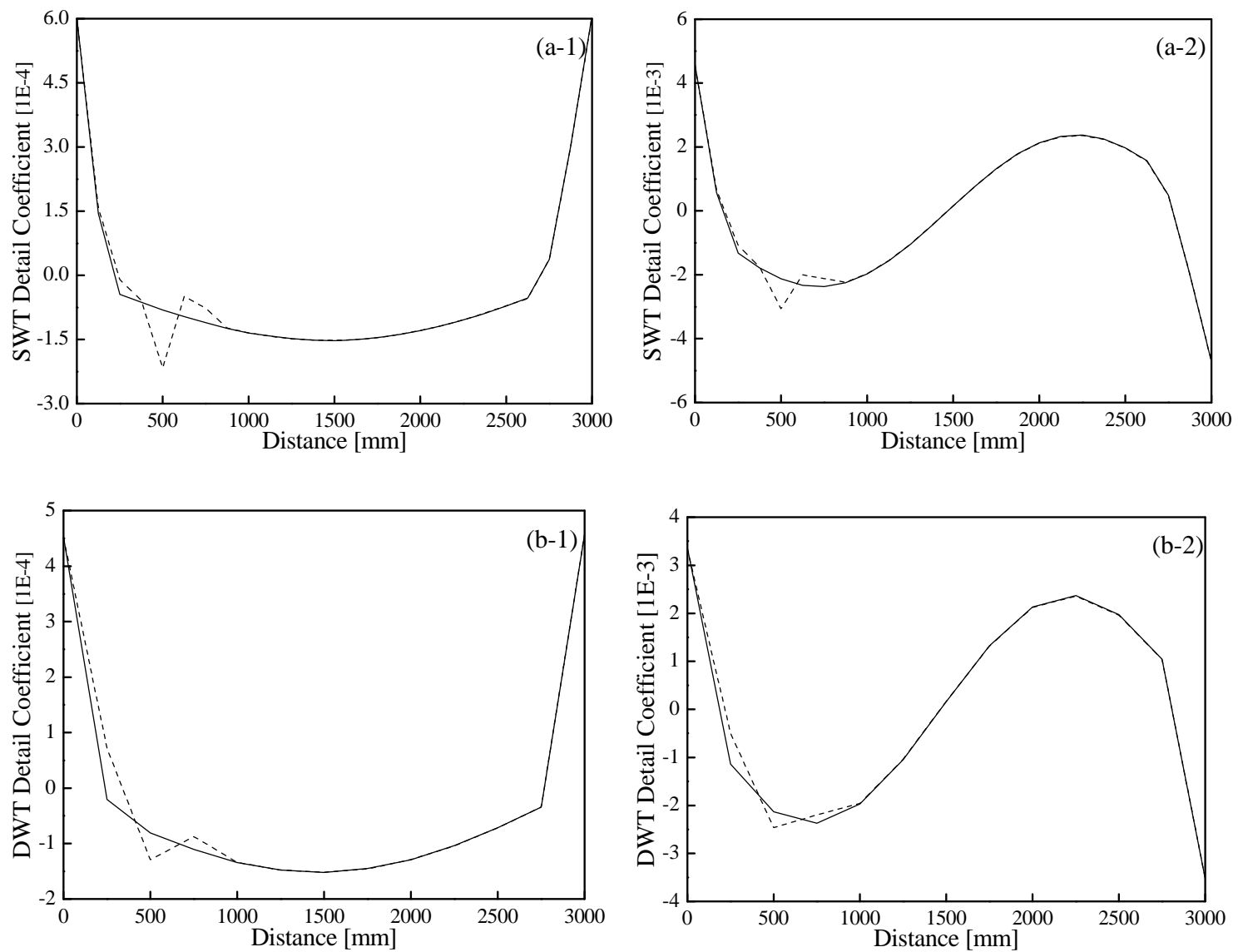

Fig.8 Comparison of SWT and DWT decomposition detail coefficients of the first two mode shapes of cracked beams $W_{c}=0.1 \mathrm{~mm}, H_{c}=1 \mathrm{~mm}$ : SWT or DWT decomposition detail coefficient of intact beam, - - - - SWT or DWT decomposition detail coefficient of cracked beams: (a-1) SWT detail of $1^{\text {st }}$ mode; (a-2) SWT detail of $2^{\text {nd }}$ mode; (b-1)DWT detail of $1^{\text {st }}$ mode; (b-2) DWT detail of $2^{\text {nd }}$ mode. 

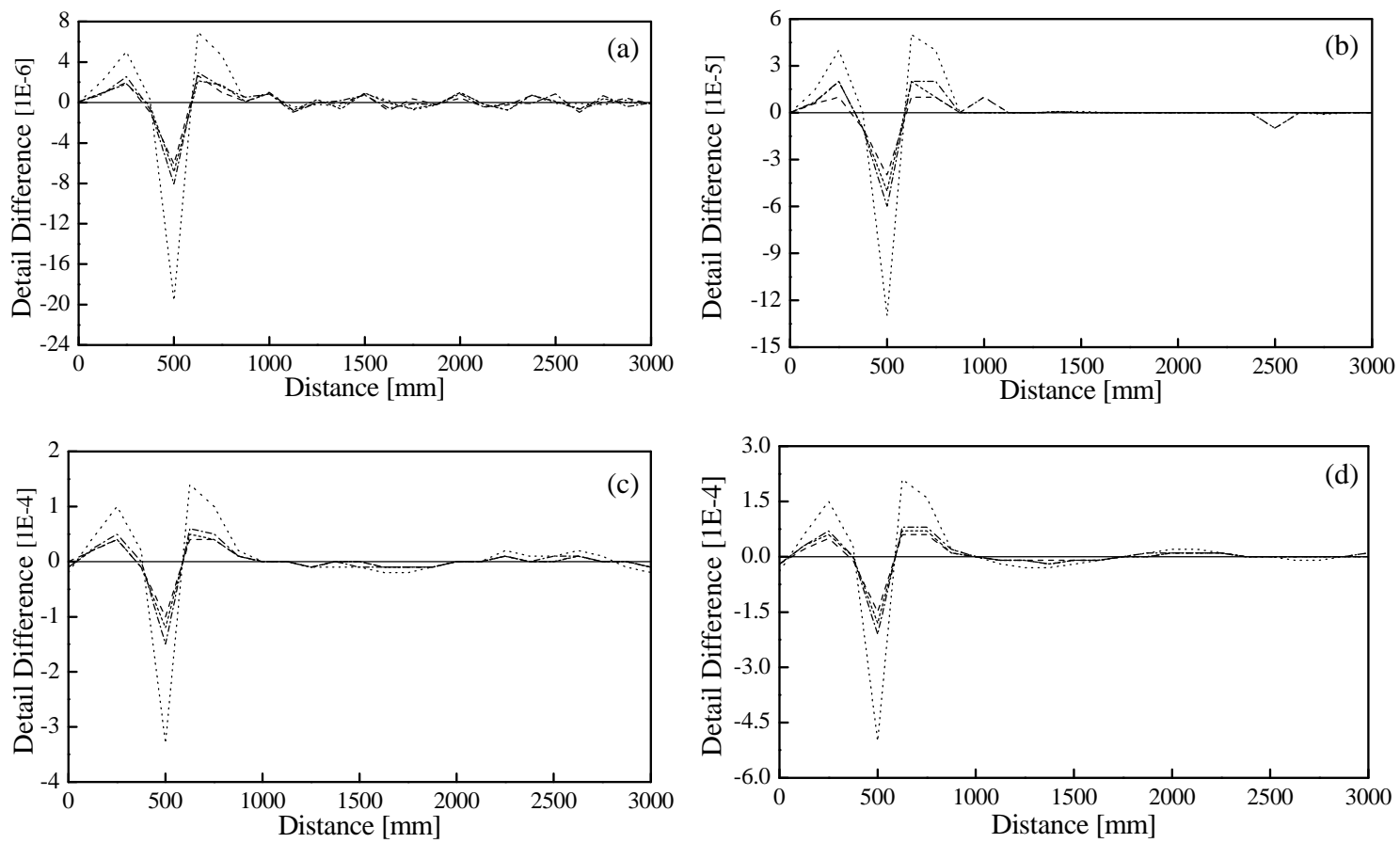

Fig.9 SWT decomposition detail coefficient difference between intact and cracked beams $H_{c}=1 \mathrm{~mm}, x_{S_{i}}^{(r)}=125 \mathrm{~mm}$ : Intact, - - - $W_{c}=0.1 \mathrm{~mm}$, $W_{c}=0.5 \mathrm{~mm},-.-.-W_{c}=1 \mathrm{~mm}$, $W_{c}=5 \mathrm{~mm}$; SWT detail difference of (a) $1^{\text {st }}$ mode shape; (b) $2^{\text {nd }}$ mode shape; (c) $3^{\text {rd }}$ mode shape; (d) $4^{\text {th }}$ mode shape. 

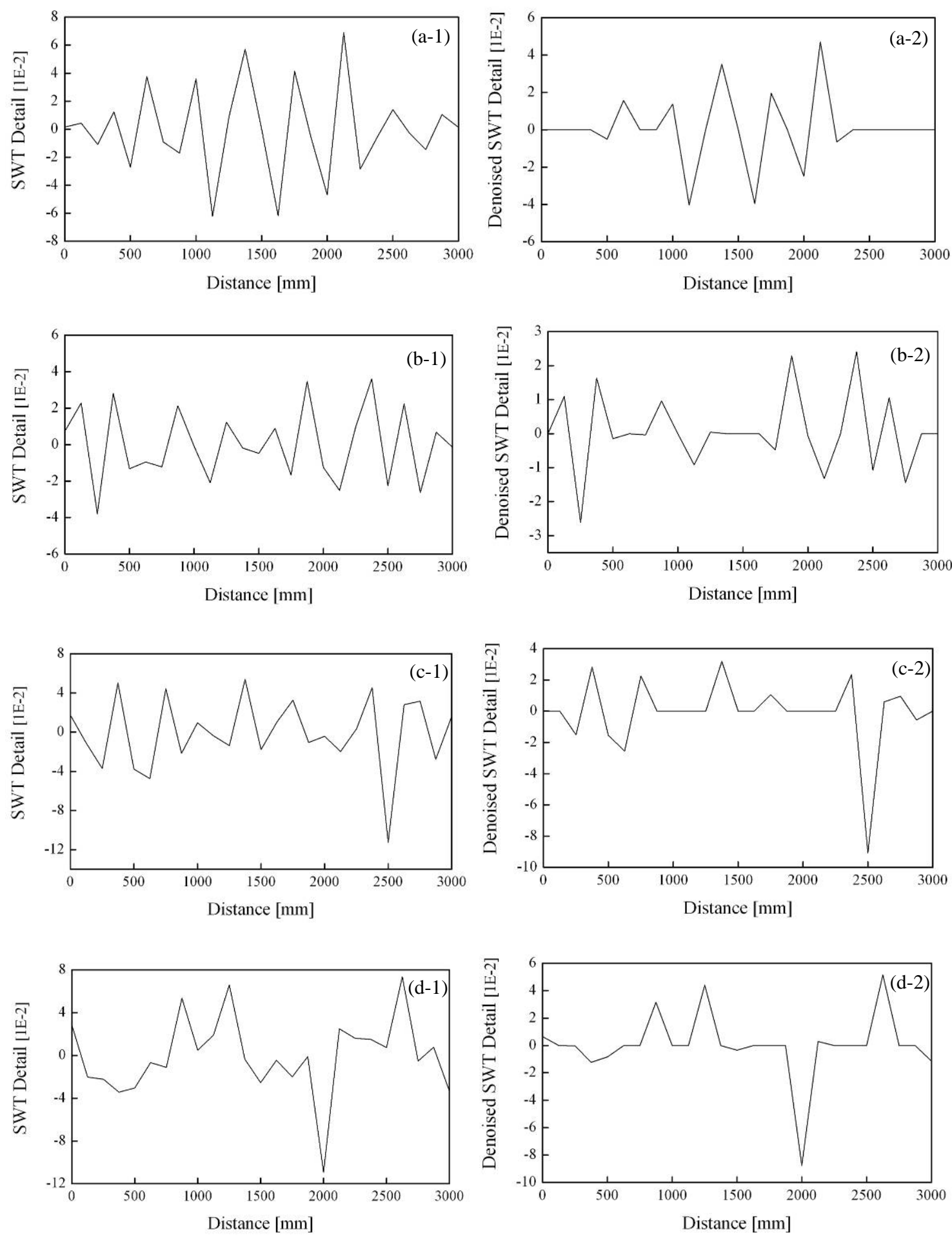

Fig.10. SWT detail coefficients (left) of the first four noisy mode shape of a cracked beam $W_{c}=1 \mathrm{~mm}, H_{c}=1 \mathrm{~mm}, \quad x_{S_{i}}^{(r)}=125 \mathrm{~mm}$ and de-noised results (right) when $10 \%$ noise is added; (a-1) to (d-1): SWT detail coefficients of $1^{\text {st }}, 2^{\text {nd }}, 3^{\text {rd }}$ and $4^{\text {th }}$ mode shapes; (a-2) to (d-2): De-noised SWT detail coefficient of $1^{\text {st }}, 2^{\text {nd }}, 3^{\text {rd }}$ and $4^{\text {th }}$ mode shapes. 

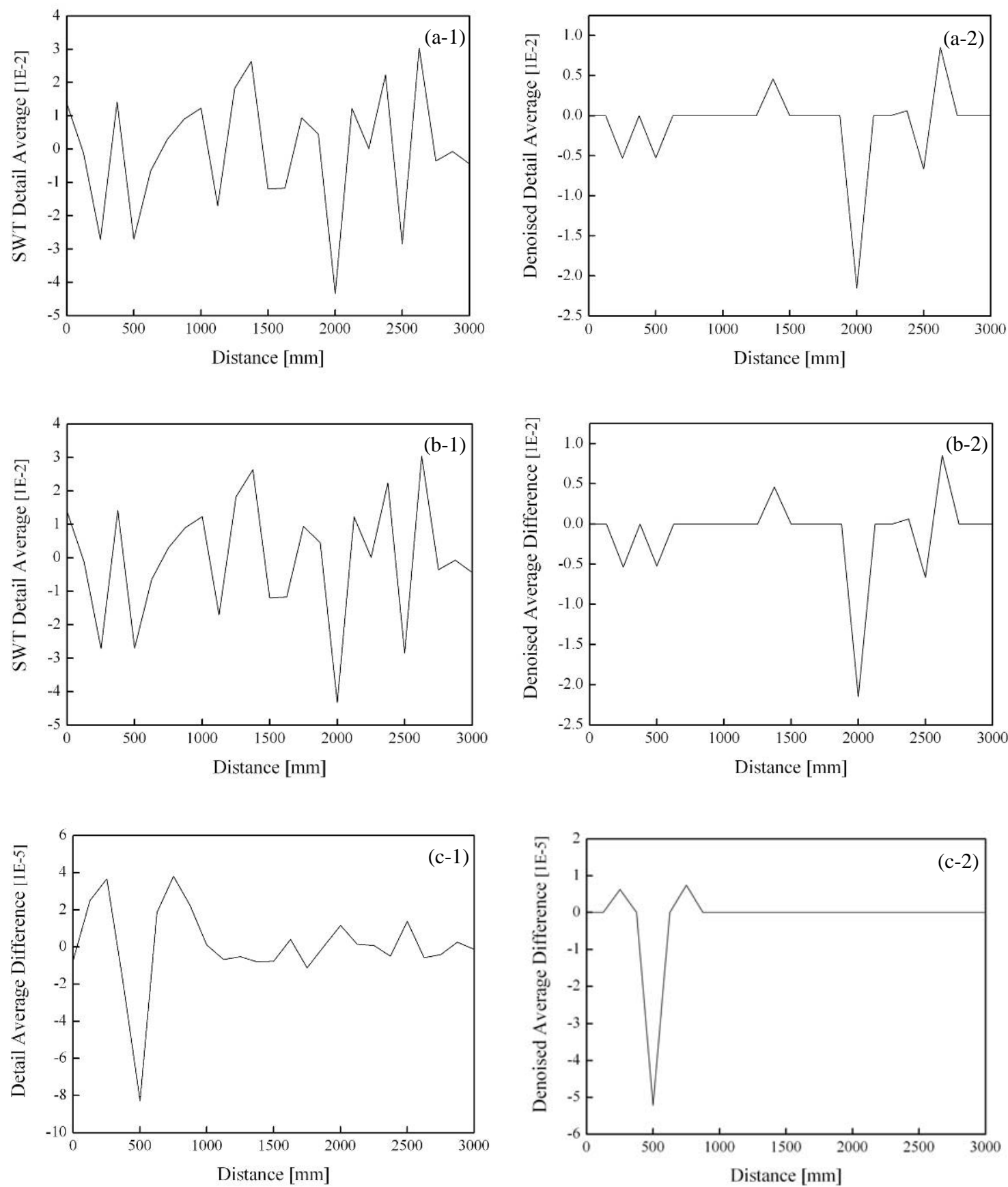

Fig.11 The average difference of the SWT detail between an intact beam and a cracked beam $W_{c}=1 \mathrm{~mm}, H_{c}=1 \mathrm{~mm}, x_{S_{i}}^{(r)}=125 \mathrm{~mm}$ : (a-1) SWT detail average of first four mode shapes of cracked beam; (a-2) De-noised SWT detail average of cracked beam; (b-1) SWT detail average of first four mode shapes of intact beam; (b-2) De-noised SWT detail average of the intact beam; (c-1) Average difference of SWT detail of first four mode shapes of cracked and intact beams; (c-2) De-noised average difference of SWT detail of cracked and intact beams. 


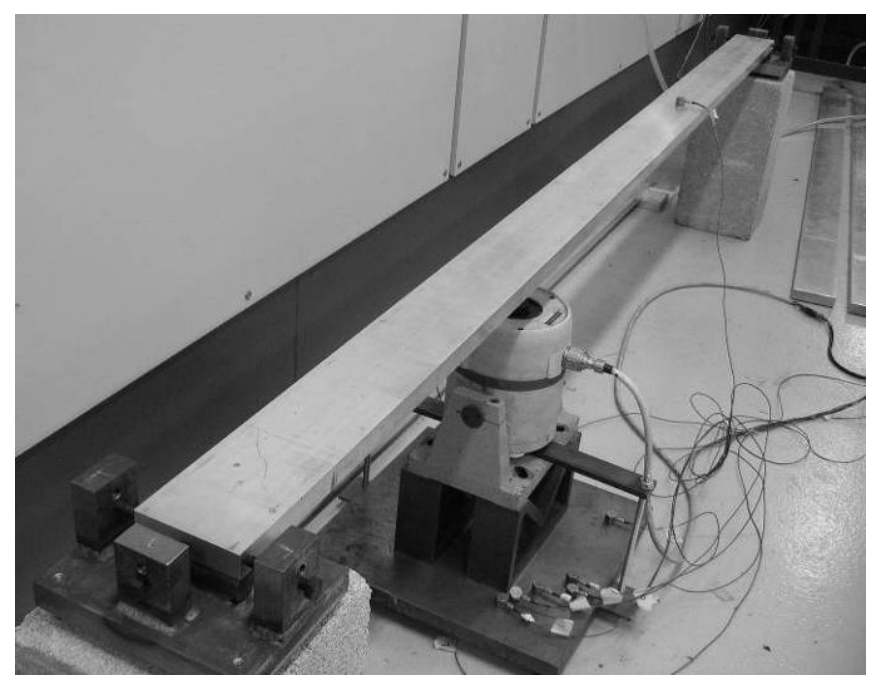

Fig.12 Experimental Setup 

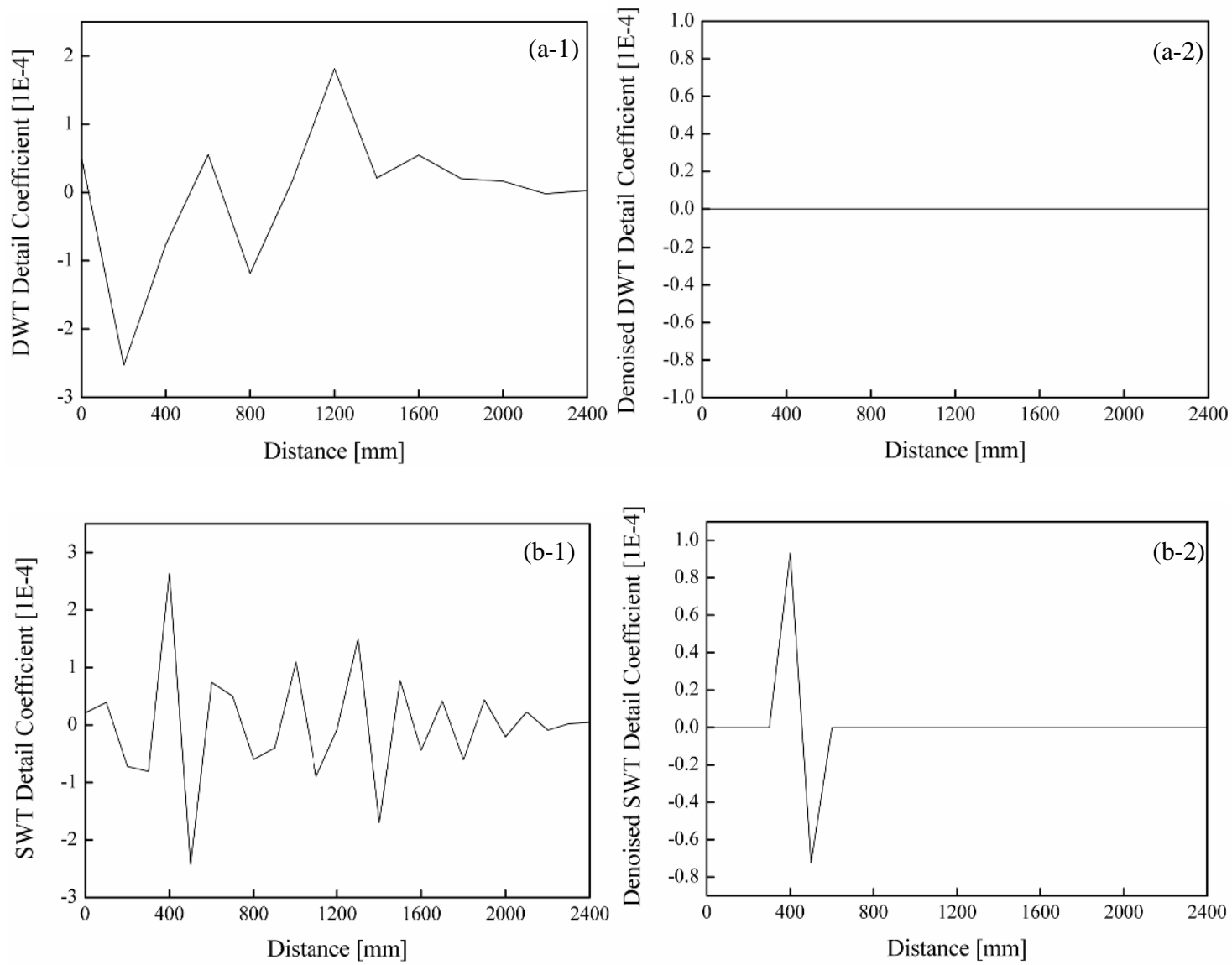

Fig.13 Comparison of SWT and DWT decomposition detail coefficients of the first mode shape of cracked aluminum beam $\left(H_{c}=2.5 \mathrm{~mm}, l_{c}=0.4 \mathrm{~m}\right)$ : (a-1) DWT decomposition detail coefficient, (a-2) Denoised DWT decomposition detail coefficient, (b-1) SWT decomposition detail coefficient, (B-2) Denoised SWT decomposition detail coefficient 